\title{
Nucleotide-induced folding of cell division protein FtsZ from Staphylococcus aureus
}

\author{
Sonia Huecas ${ }^{1}$, Alejandro J. Canosa-Valls ${ }^{1}$, Lidia Araújo-Bazán ${ }^{1}$, Federico M. \\ Ruiz $^{1}$ Douglas V. Laurents ${ }^{2}$, Carlos Fernández-Tornero ${ }^{1}$, and José M. Andreu ${ }^{1}$ \\ ${ }^{1}$ Centro de Investigaciones Biológicas CSIC, Ramiro de Maeztu 9, 28040 Madrid, Spain \\ 2 "Rocasolano" Institute for Physical Chemistry CSIC, Serrano 119, 28006 Madrid, Spain
}

Correspondence: C.F. Tornero and J.M. Andreu' Centro de Investigaciones Biológicas CSIC, Ramiro de Maeztu 9, 28040 Madrid. E-mail: cftornero@cib.csic.es; j.m.andreu@cib.csic.es

\section{Running title: FtsZ folding is induced by nucleotide binding}

Abbreviations: Apo-FtsZ, nucleotide devoid FtsZ protein; DMSO, dimethylsulfoxide; CD, circular dichroism; CTD, C-terminal domain; DSS, 4,4-dimethyl-4-silapentane-1-sulfonate; EcFtsZ, FtsZ from Escherichia coli; Ficoll 70, poly(sucrose-co-epichlorhydrin), average molecular weight 70000; $\mathrm{GdnCl}$, guanidinium chloride; GMPCP, guanosine-5'-[( $\alpha, \beta)$ methyleno]diphosphate; GMPCPP, guanosine-5' -[( $\alpha, \beta)$-methyleno]triphosphate; ITC, isothermal titration calorimetry; mant-GTP, 2'/3'-O-(N-Methyl-anthraniloyl)-guanosine-5' -triphosphate; MjFtsZ, FtsZ from Methanococcus jannaschii; MPD, 2-methyl-2,4-pentanediol; NBD, nucleotide binding domain; NBS, nucleotide binding site; NMR, nuclear magnetic resonance; ORF, open reading frame; PEG 5000 MME, polyethyleneglycol 5000 mono-methyl ether; SaFtsZ, FtsZ from Staphylococcus aureus; SaFtsZ $Z_{\mathrm{f}}$, full length SaFtsZ; SaFtsZ $\mathrm{T}_{\mathrm{T}}$, truncated SaFtsZ (residues 12-316); SaFtsZ $\mathrm{wt}_{\mathrm{wt}}$, untagged SaFtsZ; TMAO, trimethyamine N-oxide.

Protein Data Base entries: 6RVM, 6RVN, 6RVP, 6RVQ, 6SI9

Keywords: FtsZ, bacterial division, protein folding, osmolytes, GTP and inhibitors binding, ITC, fluorescence anisotropy, NMR, X-ray crystallography

Conflict of Interest: The authors declare no conflicts of interest with the contents of this article 


\section{ABSTRACT}

The essential bacterial division protein FtsZ uses GTP binding and hydrolysis to assemble into dynamic filaments that treadmill around the Z-ring, guiding septal wall synthesis and cell division. FtsZ is a structural homolog of tubulin and a target for discovering new antibiotics. Here, using FtsZ from the pathogen S. aureus (SaFtsZ), we reveal that, prior to assembly, FtsZ monomers require nucleotide binding for folding; this is possibly relevant to other mesophilic FtsZs. Apo-SaFtsZ is essentially unfolded, as assessed by NMR and CD. Binding of GTP ( $\geq$ $1 \mathrm{mM}$ ) dramatically shifts the equilibrium towards the active folded protein. Supportingly, SaFtsZ refolded with GDP crystallizes in a native structure. Apo-SaFtsZ also folds with $3.4 \mathrm{M}$ glycerol, enabling high-affinity GTP binding ( $\mathrm{K}_{\mathrm{D}} 20 \mathrm{nM}$ determined by ITC) similar to thermophilic stable FtsZ. Other stabilizing agents that enhance nucleotide binding include ethylene glycol, TMAO, and several bacterial osmolytes. High salt stabilizes SaFtsZ without bound nucleotide in an inactive twisted conformation. We identified a cavity behind the SaFtsZGDP nucleotide-binding pocket that harbors different small compounds, which is available for extended nucleotide-replacing inhibitors. We have devised a competition assay to detect any inhibitors that overlap the nucleotide site of SaFtsZ, or Escherichia coli FtsZ, employing osmolyte-stabilized apo-FtsZs and the specific fluorescence anisotropy change of mant-GTP upon dissociation from the protein. This robust assay provides a basis to screening for high affinity GTP-replacing ligands, which combined with structural studies and phenotypic profiling should facilitate development of a next generation of FtsZ-targeting antibacterial inhibitors.

\section{Introduction}

FtsZ is a cytoskeletal GTPase that forms a midcell ring-like structure (Z-ring) directing the cell division machinery in most bacteria and many archaea $[1,2]$. FtsZ forms dynamic polymers that treadmill around the Z-ring at a GTPase dependent rate, guiding septal cell wall synthesis at the division site [3-6]. The conserved tubulin-like core structure of FtsZ consists of an N-terminal nucleotide binding domain (NBD) and a C-terminal (CTD) GTPase-activating domain, joined by a long core helix. FtsZ assembles head-to-tail forming polar protofilaments in which the NBD of one monomer closely contacts the CTD of the next monomer [7, 8], so that GTP gets buried at the interface and is hydrolyzed to trigger filament disassembly. Coupled to the longitudinal association of FtsZ monomers, the lateral cleft between both FtsZ domains switches between an open conformation in assembled FtsZ and a closed conformation in unassembled monomers, which explains the cooperative assembly of single stranded protein filaments and treadmilling with GTP hydrolysis [9, 10]. The FtsZ core structure is followed by a flexible C-terminal tail 
FtsZ folding is induced by nucleotide binding consisting of a variable intrinsically disordered linker and a conserved motif for interaction with membrane tethering and regulatory proteins, as well as for protofilament bundling [11-13].

FtsZ is an attractive target, still clinically unexplored, for developing new antibiotics [14], which are required to fight antibiotic-resistant bacteria [15] including methicillin-resistant Staphylococcus aureus [16]. Previous results have shown that inhibitor binding into the interdomain cleft [14] or at the nucleotide binding site (NBS) [17, 18] can selectively target FtsZ without inhibiting eukaryotic tubulin. The effective anti-staphylococcal inhibitor PC190723 [14] binds into the open cleft, allosterically stabilizes FtsZ filaments [8, 19, 20] and is synergistic with $\beta$-lactams $[16,21]$. A new analog TXA6101 can overcome PC190723-resistant mutations [22]. We focus here on the NBS, where several well characterized FtsZ inhibitors effectively replace GTP, impair FtsZ assembly and bacterial division. However, they either are scarcely available [23] or have a limited antibacterial activity restricted to Gram-positive pathogens [18, 24]. Discovering new, chemically diverse, GTP-replacing FtsZ antibacterial inhibitors has become the next challenge. For comparison, although GTP binding and hydrolysis are key to the function of both FtsZ and tubulin, no inhibitors targeting the tubulin NBS have been reported among 94 structurally characterized compounds and anticancer drugs binding to six different sites [25].

To detect the binding of GTP-replacing ligands, competition binding assays with nucleotide-less apo-FtsZ and a labeled nucleotide can be employed. This was first accomplished using stable FtsZ from the thermophile Methanococcus jannaschii from which the native nucleotide had been separated using a low concentration of denaturant (apo-MjFtsZ) and substituting it with the fluorescent derivative mant-GTP [26, 27]. Thermophilic MjFtsZ unfolds reversibly with denaturant according to a two-state equilibrium and spontaneously refolds at 25 ${ }^{\circ} \mathrm{C}$ without the need of GTP [28]. However, FtsZ from the Gram-negative mesophile Escherichia coli (EcFtsZ) unfolds with denaturant in two steps, the first of which entails nucleotide release $[28,29]$ and the apo-protein is unstable [26]. We employed mesophilic FtsZ from Gram-positive Bacillus subtilis (BsFtsZ), purified without nucleotide, to measure binding of synthetic inhibitors $[18,24]$. Nevertheless, methods to directly screen for inhibitors of FtsZ from pathogenic $S$. aureus and from Gram-negative bacteria are required.

We observed that untagged FtsZ from methicillin-resistant S. aureus (SaFtsZ), purified with two different procedures, assembled less efficiently than other FtsZs, requiring PC190723 or the slowly hydrolyzable nucleotide GMPCPP to stabilize polymers [9, 10, 19]. We noticed, 
FtsZ folding is induced by nucleotide binding

however, that His-tagged SaFtsZ assembled readily in other studies [8]. Previous examples of assembly refractory states in other FtsZs hinted to a possible loss of bound nucleotide during purification, including EcFtsZ [30], FtsZ from Mycobacterium tuberculosis [31] and from Caulobacter crescentus [32]. An apo-SaFtsZ crystal structure was reported different from any other FtsZ structures [20]. These observations prompted us to investigate the role of nucleotide binding in FtsZ folding and to stabilize apo-SaFtsZ for screening purposes.

Here we show how SaFtsZ has a marginal stability, so that it completely unfolds in solution without nucleotide and nucleotide binding strikingly drives refolding. However, nucleotide-devoid SaFtsZ crystallizes in a distinct structure. Osmolytes stabilize folded apoSaFtsZ and enable high affinity nucleotide binding.

We have identified a cavity next to the nucleotide binding pocket that conspicuously binds small compounds and have adapted a fluorescence anisotropy assay for screening antibacterial inhibitors binding into the stabilized apo-FtsZ nucleotide site.

\section{Results}

SaFtsZ without a bound nucleotide unfolds in solution. While setting up new purification procedures for SaFtsZ, we observed that SaFtsZ spontaneously released bound nucleotide during purification and assembled poorly, whereas the protein purified in the presence of GDP was more active in assembly assays. We proceeded to initially study SaFtsZ in four forms: full length affinity purified GDP-bound protein $\left(\mathrm{SaFtsZ}_{\mathrm{f}}\right.$, with 3 N-terminal extra residues, GSH-1-390), untagged full length GDP-bound (SaFts $Z_{\mathrm{wt}}$ ) as control, the truncated GDP-bound structured core (SaFtsZ $\left.\mathrm{T}_{\mathrm{T}}, \mathrm{GSHM}-12-316\right)$ and truncated protein devoid of bound nucleotide (apo-SaFtsZ $\mathrm{T}_{\mathrm{T}}$, also GSHM-12-316; see Methods). The three nucleotide-bound versions have very similar circular dichroism (CD) spectra with one ellipticity maximum at $194 \mathrm{~nm}$ and two minima at 210 and 220 $\mathrm{nm}\left([\theta]_{222}(\right.$ holo $\left.)=-9400\right)$, indicative of folded $\alpha / \beta$ proteins with similar average secondary structures. In contrast, we found that apo-SaFts $Z_{\mathrm{T}}$ has only one minimum near $200 \mathrm{~nm}$ (Figure $\mathbf{1 A}$, black line; $[\theta]_{200}($ apo $\left.)=-15000\right)$ characteristic of apparently disordered polypeptide chains [33], indicating that both protein domains are predominantly unfolded. Analytical ultracentrifugation results showed that both $\mathrm{SaFts}_{\mathrm{f}}$ and $\mathrm{SaFts}_{\mathrm{T}}$ sediment at $\mathrm{s}_{20, \mathrm{w}}=2.8 \mathrm{~S}$ as monomeric FtsZ [13], whereas apo-SaFtsZ $\mathrm{T}_{\mathrm{T}}$ sediments more slowly at $2.0 \mathrm{~S}$, as expected for an expanded unfolded monomer (Figure 1A, inset). 
FtsZ folding is induced by nucleotide binding

We further assessed the conformation of apo-SaFtsZ $\mathrm{T}_{\mathrm{T}}$ by NMR spectroscopy. The downfield region of the $1 \mathrm{D}{ }^{1} \mathrm{H}$ NMR spectra is devoid of signals expected for the amide ${ }^{1} \mathrm{HN}$ nuclei (> 7.7 ppm) (Figure 1B, black line; the complete spectra are shown in Fig. S1). The originally protonated sample had been exchanged into $100 \% \mathrm{D}_{2} \mathrm{O}$ buffer and incubated on ice for two hours before recording this spectrum. Despite this short time in $\mathrm{D}_{2} \mathrm{O}$, essentially all the ${ }^{1} \mathrm{HN}$ signals have disappeared due to H/D exchange, which evidences a lack of stable main chain $\mathrm{H}$ bonds [34]. The peaks between 7.4 - $7.2 \mathrm{ppm}$ in the absence of GTP arise from Phe aromatic ring $\mathrm{H}$ in unfolded environments. In the absence of nucleotide, the ${ }^{1} \mathrm{H}$ NMR peaks from the methyl groups of Ile, Val and Leu resonate in a narrow band spanning 0.8 to $1.0 \mathrm{ppm}$ (Figure 1C). The chemical shift $(\delta)$ values of the Phe, Ile, Val and Leu side chain ${ }^{1} \mathrm{H}$ resemble those observed in short unstructured peptides [35]; this strongly suggests that apo-SaFts $\mathrm{Z}_{\mathrm{T}}$ is unfolded.

In contrast, the ${ }^{1} \mathrm{H}$ NMR spectra of the nucleotide bound forms SaFtsZ $Z_{\mathrm{T}}$ and $\mathrm{SaFts}_{\mathrm{f}}$ showed distinctive ${ }^{1} \mathrm{H}$ signals that evince a folded hydrophobic core (Figure 1B and 1C, green and red lines respectively, and Fig. S1). The somewhat broader and some distinct signals for the full length protein can be attributed to its larger size and longer correlation time as well as to the presence of disordered $\mathrm{N}$ and $\mathrm{C}$-terminal segments. In addition, ${ }^{1} \mathrm{H}$ peaks with $\delta$ values of 7.8 $9.8 \mathrm{ppm}$ are seen which arise from amide ${ }^{1} \mathrm{HN}$. Since these peaks would have exchanged out for $\mathrm{D}$ and become invisible without protective intra-protein $\mathrm{H}$-bonds, this is indicative of stable secondary structure. This evidence for secondary and tertiary structure strongly suggests that both SaFtsZ $\mathrm{T}_{\mathrm{T}}$ and SaFtsZ $\mathrm{f}$ are folded in the presence of nucleotide at $25{ }^{\circ} \mathrm{C}$ in $\mathrm{D}_{2} \mathrm{O}$. Finally, we recorded a series of $1 \mathrm{D}{ }^{1} \mathrm{H}$ NMR spectra on these samples at temperatures ranging from 5.0 to $45.0{ }^{\circ} \mathrm{C}$ (Fig. S2). Based on the observation of protected ${ }^{1} \mathrm{HN}$ signals and broad signal dispersion, it can be concluded that both $\mathrm{SaFts}_{\mathrm{T}}$ and $\mathrm{SaFts}_{\mathrm{f}}$ are folded over the complete range of temperatures. The spectral quality appears to improve at higher temperatures due to shorter correlations times.

Apo-SaFtsZ $\mathrm{T}_{\mathrm{T}}$ undergoes slow nucleotide-induced assembly. We compared the four different SaFtsZ forms in polymerization assays. Monitoring with light scattering the polymer formation by SaFtsZ with $50 \mu \mathrm{M}$ GMPCPP and $10 \mathrm{mM} \mathrm{MgCl}_{2}$, and polymer disassembly upon consumption of the slowly hydrolysable GTP analog, revealed kinetic differences between them (Figure 2A), including weaker assembly without C-terminal tail [13]. Remarkably, apo-SaFts $Z_{\mathrm{T}}$ becomes functional with nucleotide, but assembles more slowly only after a long lag time ( 90 min with GMPCPP, $\sim 150$ min with GTP), which could be related to rate-limiting monomer folding or polymer nucleation events. Polymer sedimentation assays with GMPCPP/GMPCP, 
FtsZ folding is induced by nucleotide binding GTP/GDP and mant-GTP/mant-GDP indicated that the three guanosine triphosphates, but not GDP, specifically induce SaFtsZ assembly (Figure 2B). Electron microscopy showed that $\mathrm{SaFts}_{\mathrm{f}}$ forms curved protofilaments coalescing into spirals or bundles whereas both $\mathrm{SaFts}_{\mathrm{T}}$ and apo-SaFtsZ $\mathrm{Z}_{\mathrm{T}}$ form more straight bundles of protofilaments (Figure 2C) as expected [13]. We inferred from these results that unfolded apo-SaFtsZ folds into an actively assembling native species upon nucleotide binding.

Osmolytes and ionic strength stabilize folded apo-SaFtsZ $\mathbf{Z}_{\mathbf{T}}$ in solution. We found that apo$\mathrm{SaFtsZ}_{\mathrm{T}}$ acquires a CD spectrum similar to the native protein in the presence of glycerol (3.4 M, $25 \% \mathrm{v} / \mathrm{v}$ ), a widely employed protein stabilizing co-solvent [36, 37]. Titration of the glycerol effect shows a gradual spectral change with an isosbestic point around $207 \mathrm{~nm}$, suggesting linear combinations of the unfolded and folded protein spectra (Figure 3A). The ellipticity values at $222 \mathrm{~nm}$ (a characteristic $\alpha$-helical CD band) as a function of the stabilizer concentration (inset) can be accurately fit by a two-state equilibrium between the unfolded and folded protein [38, 39] (Methods). According to this model, the free energy change of folding depends linearly on osmolyte concentration:

$\Delta \mathrm{G}_{\text {fold,obsd }}=\Delta \mathrm{G}_{\text {fold, } 0 \mathrm{M} \text { glycerol }}-m$ [glycerol $]$

where $\Delta \mathrm{G}_{\text {fold }}, 0 \mathrm{M}$ glycerol $=2.6 \pm 0.2 \mathrm{kcal} \mathrm{mol}^{-1}$ and the osmolyte efficacy is $m=1.3 \pm 0.1 \mathrm{kcal} \mathrm{mol}^{-}$ ${ }^{1} \mathrm{M}^{-1}$. Notice that folded apo-SaFtsZ is unstable in buffer (unfavorable $\Delta \mathrm{G}_{\text {fold }}>0$ ), there is a point at $2 \mathrm{M}$ glycerol at which $\Delta \mathrm{G}_{\text {fold }}=0$, and the folded protein becomes stabilized (with favorable $\Delta \mathrm{G}_{\text {fold }}<0$ ) at higher glycerol concentrations. Thus $\Delta \mathrm{G}_{\text {fold, } 3.4 \mathrm{M} \text { glycerol }}=-1.8 \mathrm{kcal} / \mathrm{mol}$, which is $4.4 \mathrm{kcal} / \mathrm{mol}$ more favorable than in the absence of glycerol.

Apo-SaFtsZ $Z_{\mathrm{T}}$ also acquires secondary structure upon increasing ionic strength from 50 $\mathrm{mM} \mathrm{KCl}$ to $450 \mathrm{mM} \mathrm{KCl}$ (Fig. S3); a two-state model fit indicates $\Delta \mathrm{G}_{\text {fold }}, 0 \mathrm{M} \mathrm{KCl}=3.6 \pm 0.8 \mathrm{kcal} /$ mol, similar within error to the glycerol result. However, in contrast with glycerol, high $\mathrm{KCl}$ concentrations strongly reduce apo-SaFtsZ's nucleotide binding affinity, as will be shown later. $\mathrm{NaCl}$ had similar $\mathrm{CD}$ effects as $\mathrm{KCl}$. Changing the temperature from $25^{\circ} \mathrm{C}$ to $5{ }^{\circ} \mathrm{C}$ or $37{ }^{\circ} \mathrm{C}$ or employing three different $\mathrm{Mg}^{2+}$-containing FtsZ assembly buffers did not induce significant folding (Fig. S4A). The macromolecular crowding agent PEG5000 MME (20\% w/v, employed for $\mathrm{SaFtsZ}_{\mathrm{T}}$ crystallization) induced a glycerol-like CD spectrum of apo-SaFts $\mathrm{T}_{\mathrm{T}} \mathrm{CD}$ but $20 \%$ w/v Ficoll70 did not, suggesting a lack of a pure excluded volume effect on folding (Fig. S4A). 2-methyl-2,4-pentanediol (MPD, 20\% v/v), a popular crystallization additive, induced a different CD spectrum with a negative band at $216 \mathrm{~nm}$ characteristic of $\beta$-sheet (Fig. S4B). 
FtsZ folding is induced by nucleotide binding

Interestingly, different protein stabilizing osmolytes induce apo-SaFts $Z_{\mathrm{T}}$ folding, in the order of effectivity: glycerol $(25 \% \mathrm{v} / \mathrm{v}) \approx \mathrm{TMAO}$ (trimethyamine $\mathrm{N}$-oxide, $1 \mathrm{M})>$ trehalose $(1 \mathrm{M})$ $>$ ethylene glycol $(20 \% \mathrm{v} / \mathrm{v})>$ betaine $(1 \mathrm{M}) \approx$ proline $(1 \mathrm{M})$ (Fig. S4B). These results collectively point to osmophobic stabilization of the folded protein relative to the unfolded state, in which the peptide backbone experiences unfavorable interactions with osmolyte [38, 40].

Nucleotide-induced folding of apo-SaFts $Z_{\mathbf{T}}$. We observed that apo-SaFtsZ $\mathrm{T}_{\mathrm{T}}$ progressively acquires a native-like $\mathrm{CD}$ spectrum with increasing concentrations of the natural ligand GTP (Figure 3B). Analogously to glycerol, there is an isosbestic point near $206 \mathrm{~nm}$, suggesting a mixture of unfolded and folded (GTP-bound) FtsZ molecules in the solution. The minimum at $210 \mathrm{~nm}$ is slightly more marked with GTP than with glycerol. Attainment of equilibrium CD spectra following GTP addition required about 40 minutes, indicating slow binding or a previous rate-limiting folding step, which could explain the slow nucleotide-induced assembly observed

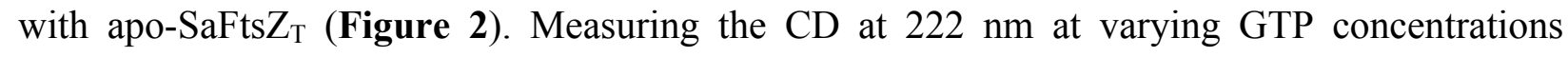
indicated low apparent affinity GTP binding (notice that the CD change measures protein folding rather than the ligand binding itself). This is not surprising, considering that the unfavorable folding free energy $\left(\Delta \mathrm{G}_{\text {fold }}\right.$, $0 \mathrm{M}$ glycerol $\left.=2.6 \mathrm{kcal} / \mathrm{mol}\right)$ opposes binding. Since part of the binding energy is "spent" folding the protein, the binding affinity is actually higher than that observed experimentally. Approximately fitting a simple ligand-facilitated folding mechanism to the CD change (Figure 3B inset) permitted us to extract an estimate of the free energy change of GTP binding to the folded protein $\Delta \mathrm{G}_{2}=-9.4 \pm 0.2 \mathrm{kcal} / \mathrm{mol}$. Both $1 \mathrm{mM}$ GTP or GDP induced folding of apo-SaFtsZ $\mathrm{T}_{\mathrm{T}}$ to similar extents, however the degree of folding with $1 \mathrm{mM}$ mant-GTP was less complete as monitored by CD.

Monitoring SaFtsZ $\mathrm{T}_{\mathrm{T}}$ folding by $1 \mathrm{D}{ }^{1} \mathrm{H}$ NMR has shown that upon addition of GTP to apo$\mathrm{SaFtsZ}_{\mathrm{T}}$, the aliphatic methyl groups' band at 0.8 to $1.0 \mathrm{ppm}$ decreases in intensity with time, while shoulders and peaks with chemical shift values $<0.8 \mathrm{ppm}$ arise (Fig. S5). These observations are consistent with methyl groups in distinct magnetic environments near aromatic rings in the hydrophobic core of folding SaFts $\mathrm{Z}_{\mathrm{T}}$. The observed kinetics of refolding induced by GTP can be fit by a first order reaction with an observed rate of $0.017 \pm 0.002 \mathrm{~min}^{-1}$, i.e. a kinetic lifetime of $\sim 60$ minutes (Figure 3C). Regarding the downfield region of the $1 \mathrm{D}{ }^{1} \mathrm{H}$ NMR spectrum, after adding GTP, the peaks between 7.4 - 7.2 ppm arising from Phe aromatic ring $\mathrm{H}$ in unfolded environments weaken and new peaks indicative of Phe aromatic ring $\mathrm{H}$ in folded conformers appear at $7.1-6.7 \mathrm{ppm}$. The appearance of peaks at $6.10,5.75,5.56,5.44,5.17 \mathrm{ppm}$ which can be tentatively assigned to ${ }^{1} \mathrm{H} \alpha$ in folded $\beta$-sheets, is also noteworthy (Fig. S5). Note 
that the intensity of the $1.00-0.80 \mathrm{ppm}$ band does not reach zero at equilibrium as Val, Ile and Leu methyl groups far from aromatic groups in the folded protein still resonate there. Also the integration of the upfield $(\delta<0.75 \mathrm{ppm})$ folded peaks depends on the number of methyl groups they contain and the line broadness. Considering these two points, it can be concluded that the protein is completely folded when the peak intensity versus time reaches a stable plateau.

Nucleotide binding is enabled by FtsZ folding. Considering that guanine nucleotides induce apo-SaFts $Z_{\mathrm{T}}$ folding with low apparent affinity and that folding is also induced by osmolytes, we reasoned that osmolytes should enable high affinity nucleotide binding. We first found that glycerol enables binding of fluorescent mant-GTP by apo-SaFtsZ $\mathrm{T}_{\mathrm{T}}$. Therefore, we determined the energetics of interaction of folded apo-SaFts $\mathrm{Z}_{\mathrm{T}}$ with guanine nucleotides in $3.4 \mathrm{M}$ glycerol, employing isothermal titration calorimetry (ITC). Nucleotide binding by folded apo-SaFts $\mathrm{Z}_{\mathrm{T}}$ in glycerol is an exothermic enthalpy-driven interaction (Figure 4A and Table 1). GTP and GDP binding affinities are similar $\left(K_{D} 20 \mathrm{nM}\right)$, and the GDP affinity decreases when $\mathrm{Mg}^{2+}(10 \mathrm{mM})$ is added $\left(K_{D} 250 \mathrm{nM}\right)$. Increasing ionic strength strongly reduces the GTP binding affinity: the $K_{D}$ value increases ten-fold in $0.35 \mathrm{M} \mathrm{KCl}$ (Table 1), and about hundred-fold in $1.0 \mathrm{M} \mathrm{KCl}(1.6 \mu \mathrm{M}$, Table S1). The GTP binding affinity of apo-SaFtsZ $Z_{\mathrm{T}}$ strongly decreases at low glycerol concentration (Figure 4B; $\mathrm{K}_{\mathrm{D}} 9 \mu \mathrm{M}$ at $0.7 \mathrm{M}$ glycerol). The binding of mant-GTP to apoSaFtsZ $_{\mathrm{T}}$ was also measured by ITC $\left(\mathrm{K}_{\mathrm{D}} 200 \mathrm{nM}\right.$ in $3.4 \mathrm{M}$ glycerol, $\sim 100$-fold weaker in $0.35 \mathrm{M}$ $\mathrm{KCl}$; Figure 4C and Table 1).

In order to obtain insight into the thermodynamics of the GTP interaction with unfolded apo-SaFts $Z_{\mathrm{T}}$, including the folding and binding contributions, we made ITC measurements at varying glycerol concentrations, finding marked changes in the observed binding free energy, enthalpy and entropy (Table S2). The change in apparent affinity (Figure 4D, filled circles) has a sigmoidal shape reminiscent of the glycerol-induced folding curve (Figure 3A). Nucleotide binding could not be directly measured in the buffer without glycerol because it becomes very slow and nearly athermic. Instead, averaged measurements at low glycerol concentrations afford $\Delta \mathrm{G}_{\mathrm{b} \text {, obsd }}(\leq 0.7 \mathrm{M}$ glycerol $)=-7.0 \pm 0.4 \mathrm{kcal} / \mathrm{mol}\left(\mathrm{K}_{\mathrm{D}} \sim 7 \mu \mathrm{M}\right)$, providing a reasonable empirical estimate for the low GTP binding affinity in the absence of glycerol. The observed binding free energy change in excess glycerol is $\Delta \mathrm{G}_{\mathrm{b} \text {, obsd }}(3.4 \mathrm{M}$ glycerol $)=-10.5 \pm 0.1 \mathrm{kcal} / \mathrm{mol}$. Calculation of the GTP binding affinity to the folded protein at each glycerol concentration, according to the indicated ligand-facilitated folding model (Figure 4D, void circles), indicates a binding free energy change at low glycerol concentrations $\Delta \mathrm{G}_{2}(\leq 0.7 \mathrm{M}$ glycerol $)=-9.0 \pm 0.3 \mathrm{kcal} / \mathrm{mol}$, which is compatible with the estimate from the GTP-induced CD change $(-9.4 \pm 0.2 \mathrm{kcal} / \mathrm{mol}$, 
Figure 3B). Adopting the average value, the increment binding free energy change between buffer and glycerol solutions can thus be estimated as $\Delta \Delta \mathrm{G}_{\mathrm{b}}(\leq 0.7->3.4 \mathrm{M}$ glycerol $)=-1.3 \pm 0.3$ $\mathrm{kcal} / \mathrm{mol}$. The observed enthalpy-driven binding of GTP to apo-SaFtsZ $\mathrm{T}_{\mathrm{T}}$ in $3.4 \mathrm{M}$ glycerol strikingly changes to weakly exothermic and predominantly entropy-driven binding at low glycerol concentrations (see the thermodynamic signature plots in Figure 4E).

For comparison, we also measured the glycerol effect on GTP binding employing a close homolog apo-protein (BsFtsZ, $80 \%$ sequence identity with $\mathrm{SaFtsZ}_{\mathrm{T}}, 100 \%$ identical in the NBS) that is metastable in the absence of glycerol, obtaining a value $\Delta \Delta \mathrm{G}_{\mathrm{b}}$ Bs $(0->3.4 \mathrm{M}$ glycerol $)=-$ $2.0 \pm 0.1 \mathrm{kcal} / \mathrm{mol}$. Employing the stable thermophilic apo-MjFtsZ (41\% sequence identity with SaFtsZ $_{\mathrm{T}}$ ) permits direct measurement of nucleotide binding to a folded FtsZ [41], giving a smaller glycerol effect $\Delta \Delta \mathrm{G}_{\mathrm{b} \text { Mj }}(0->3.4 \mathrm{M}$ glycerol $)=-0.6 \pm 0.2 \mathrm{kcal} / \mathrm{mol}$ (Table S2).

Structures of nucleotide-refolded FtsZ and holo-FtsZ. We employed X-ray crystallography as a tool to investigate SaFtsZ folding and ligand binding. Refolded FtsZ crystallized following

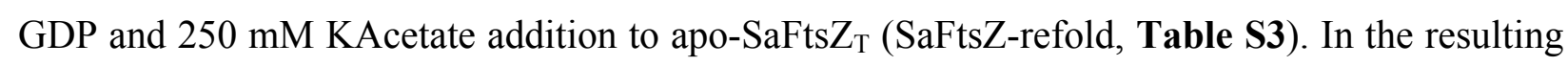
structure at $1.90 \AA$ resolution, the CTD coordinates a $\mathrm{Ca}^{2+}$ ion through the T7 loop (Figure 5AB) and SaFts $\mathrm{Z}_{\mathrm{T}}$ monomers form straight filaments with head-to-tail contacts, where the tip of the T7 loop lies next to the GDP molecule of the neighboring monomer, thus burying the nucleotide between monomers. These features are the same as in native SaFts $Z_{\mathrm{T}}$ structures with bound GDP in the open (tense) conformation, as confirmed by superposition to available structures (PDB entries 3VOA [20], 5H5G [42] and 5MN4 [10] with alpha-carbon rmsd values of 0.20, 0.26, 0.23 Å respectively).

We have also identified a cavity that is located next to the guanine moiety of the GDP molecule (Figure 5C), employing structures of GDP-bound $\mathrm{SaFtsZ}_{\mathrm{T}}$ (Table S3) at resolutions between 1.14 and $1.24 \AA$, which are the highest reported to date. This cavity is about $7 \AA$ in depth and $6 \AA$ in width (total volume $\sim 250 \AA^{3}$ ) and exhibits an overall non-charged surface. In one of the structures $\left(\mathrm{SaFts}_{\mathrm{T}}\right.$-Wat, Fig. S6), this cavity hosts 6 water molecules, one of which belongs to the solvent shell directly contacting GDP (Figure 5C). Another structure (SaFtsZ $\mathrm{T}^{-}$ EthGly, Fig. S6) shows clear density for ethylene glycol, a crystallization additive, displacing 3 water molecules from the cavity (Figure 5C) and forming hydrogen bonds with the main chain of Gly22 and the side chain of Thr102 (Fig. S6), as well as several non-polar interactions with SaFtsZ and GDP. An additional structure shows density for the co-solvent 1-methyl-2pyrrolidone, which displaces 5 of the 6 water molecules from the cavity, at a position that 
partially overlaps with that of EthGly, and establishes mostly non-polar interactions with protein residues and with the guanine moiety of GDP $\left(\right.$ SaFts $_{\mathrm{T}}-\mathrm{MetPyr}$, Fig. S6). In fact, an equivalent MetPyr molecule has been previously observed following crystal soaking in this co-solvent (PDB 5XDT; [22]). We notice that this cavity can also host MPD (PDB 5MN4; [10]; Fig. S6). These results together identify a cavity at the bottom of the nucleotide-binding pocket that accommodates several small ligands of different chemical nature.

Nucleotide dissociation in high salt rearranges its binding site and the interdomain interface. We also determined the crystal structure of $\mathrm{SaFts}_{\mathrm{T}}$ in the absence of bound nucleotide (SaFtsZ $\mathrm{T}_{\mathrm{T}}$-Apo) at $2.15 \AA$ resolution, by using $2.1 \mathrm{M}$ ammonium sulfate as precipitant (Figure 5D, Table S3). This structure that no longer forms filaments unexpectedly superposed with an available structure devoid of $\mathrm{Ca}^{2+}$ and nucleotide (Fig. S7A-C, rmsd values of 0.4 to 1.1 $\AA$ ) obtained from crystals grown under different conditions, in the presence of $1 \mathrm{M}$ lithium chloride and $0.34 \mathrm{M}$ chelating agent sodium malonate, in which loop T4 overlaps the position normally occupied by GDP and collapses the binding pocket [20].

In addition to rearrangements in the NBS, conversion from the GDP-bound to the apo form of $\mathrm{SaFts}_{\mathrm{T}}$ (this work and [20]) involves a massive reorganization of the interface between the NBD and CTD domains (Figure 5D). This twist region spans residues 166 to 221 in SaFtsZ (Figure 5A). The conformational rearrangement from the GDP-bound state to the folded apo state is better visualized as a morph movie between both crystal structures (Supp. Movie 1). In summary, helix H6 (residues 166-172) turns by $90^{\circ}$ and occupies the position of the middle part of helix $\mathrm{H} 7$ in GDP-bound $\mathrm{SaFtsZ}_{\mathrm{T}}$. In turn, the N-terminal half of $\mathrm{H} 7$ also rotates by $90^{\circ}$ to occupy the position of $\mathrm{H} 8$ in the GDP-bound form, while the $\mathrm{C}$-terminal half of $\mathrm{H} 7$ forms a new $\beta$-strand that inserts between the $\mathrm{N}$ - and $\mathrm{C}$-terminal $\beta$-sheets, connecting into a single $\beta$-sheet that traverses both domains. Finally, the T7 loop disengages from the $\mathrm{Ca}^{2+}$ ion and rotates by about $180^{\circ}$ to reach, together with $\mathrm{H} 8$, the T5 loop on the opposite side of the protein. As a result, the $\mathrm{CTD}$, which roughly retains its overall fold $\left(\mathrm{rmsd}=2.1 \AA\right.$ ), rotates by about $25^{\circ}$ with respect to its position in GDP-bound $\mathrm{SaFtsZ}_{\mathrm{T}}$ (Figures $5 \mathrm{C}$ and $\mathbf{S 7 D}$ ).

Fluorescent nucleotide binding by osmolyte-stabilized apo-FtsZ and competition assays for inhibitor screening. We asked whether protein-stabilizing additives would generally induce high affinity nucleotide binding by apo-SaFts $\mathrm{Z}_{\mathrm{T}}$. To address this question, we employed mantGTP, whose fluorescence anisotropy increases markedly by rotational immobilization upon binding to other FtsZs [18, 27, 43]. We screened different additives for specific enhancement of the anisotropy change of mant-GTP $(1 \mu \mathrm{M})$ upon addition of apo-SaFts $Z_{\mathrm{T}}(1 \mu \mathrm{M})$. We found that 
glycerol, TMAO, ethylene glycol, betaine, trehalose and L-proline enable specific mant-GTP binding (Fig. S8), in an order related to their efficiency in inducing folding as monitored by CD (Fig. S4). In contrast, MPD, PEG5000 MME and Ficoll 70 do not increase mant-GTP anisotropy (Fig. S8) in spite of the secondary structure folding by PEG5000 MME (Fig. S4). These results clearly support a general osmolyte-induced folding effect [40] stabilizing NBS in apo-SaFtsZ $Z_{\mathrm{T}}$, rather than a volume exclusion stabilizing effect. $\mathrm{KCl}$ stabilizes secondary structure (Fig. S2), but induces medium-affinity nucleotide binding in ITC (Tables 1 and S1), and cancels high affinity mant-GTP binding in fluorescence anisotropy (Fig. S8). KAcetate and KGlutamate (0.25 M) partially induce mant-GTP binding, but higher concentrations $(0.5 \mathrm{M})$ reduce binding (Fig. S8).

Titration of mant-GTP with apo-SaFtsZ $\mathrm{T}_{\mathrm{T}}$ in $3.4 \mathrm{M}$ glycerol confirmed high affinity binding (Fig. S9). A previously developed competition assay based on the decrease of mant-GTP anisotropy upon release from FtsZ $[18,27]$ now permits the measurement of any molecule's binding to the NBS of FtsZ from the pathogen S. aureus. This is made possible employing glycerol-stabilized apo-SaFtsZ $\mathrm{T}_{\mathrm{T}}$. As a proof of concept we have tested two GTP-replacing synthetic FtsZ inhibitors that bind with micromolar affinities to apo-SaFts $Z_{\mathrm{T}}$. Compound UCM44 [18] displaces mant-GTP with an $\mathrm{K}_{\mathrm{D} \text {,app }} 26 \mu \mathrm{M}$ and UCM81 (compound 2; [24])

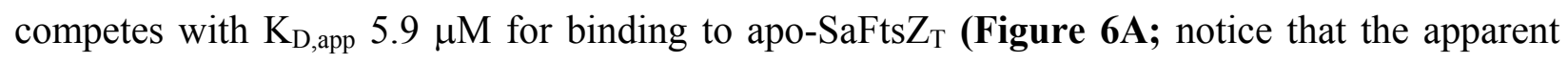
affinities of these competitors change with glycerol concentration). Finally, for additional corroboration we prepared stabilized FtsZ from E. coli predominantly devoid of nucleotide (with 0.3 residual GDP per protein molecule; see purification Methods) and performed similar mantGTP competition tests (Figure 6B). The results indicate that UCM44 and UCM81 have a reduced apparent affinity for EcFtsZ, compatible with their weaker inhibitory activity [18], and demonstrate the feasibility of testing potential inhibitors of Gram-negative FtsZ.

\section{Discussion}

Solution and crystal structures of apo-SaFtsZ $\mathrm{T}_{\mathrm{T}}$. Our CD and NMR results demonstrate that the SaFtsZ structural fold has marginal stability under usual experimental conditions; the protein structure is held together by the bound guanine nucleotide. When stripped of the nucleotide, the CD spectrum of SaFtsZ $_{\mathrm{T}}$ lacks most of the ellipticity characteristic of $\alpha$-helical and $\beta$-sheet secondary structures, resembling spectra from intrinsically disordered proteins [44]. The observations from NMR spectroscopy corroborate that apo-SaFts $Z_{\mathrm{T}}$ is unfolded. The lack of protection against amide H/D exchange detected by NMR evinces that no stable secondary structure is present. In addition, the absence of chemical shift dispersion for apo-Fts $\mathrm{T}_{\mathrm{T}} \mathrm{Phe}$ 
FtsZ folding is induced by nucleotide binding

aromatic ${ }^{1} \mathrm{H}$ and aliphatic methyl ${ }^{1} \mathrm{H}$ indicates there is no well packed hydrophobic core [45]. Upon addition of GTP, apo-SaFtsZ $\mathrm{T}_{\mathrm{T}}$ slowly refolds attaining a well-packed hydrophobic core. We were able to obtain a crystal structure of refolded FtsZ in complex with GDP that closely superimposes with the canonical native structure of SaFtsZ, thus confirming the CD and NMR solution folding results.

We also obtained a crystal structure of SaFtsZ where GDP is absent, by incubating the $\mathrm{SaFts}_{\mathrm{T}}$-GDP complex in a precipitant solution containing ammonium sulfate at mildly acidic $\mathrm{pH}$. An equivalent structure was previously obtained by crystallizing the protein in the presence of lithium chloride and the chelating agent sodium malonate [20]. Given that the two crystallization conditions are chemically different while the structures are similar, we conclude that the same folded SaFtsZ-Apo structure is possibly generated by a similar mechanism, likely due to weaker polar interactions of the nucleotide with SaFtsZ at high salt concentration. The SaFtsZ-Apo structure may thus represent an inactive conformational state, supported by the fact that both NBD and CTD undergo small rearrangements, whereas the central part of the protein dramatically twists in the transition from the GDP-bound to apo form (Supp. Movie 1). Our result suggests that this fold is a consequence of the absence of the nucleotide rather than calcium removal, supported by the native fold of one $\mathrm{Ca}^{2+}$-less GDP-bound SaFtsZ molecule [42].

Finally, it is tempting to hypothesize that the apo-SaFts $\mathrm{Z}_{\mathrm{T}}$ folding observed by $\mathrm{CD}$ upon addition of $0.5 \mathrm{M} \mathrm{KCl}$ (Results, Fig. S3) may correspond to the SaFts $Z_{\mathrm{T}}$-Apo crystal structure; this is supported by the markedly reduced nucleotide binding affinity in $\mathrm{KCl}$. However, osmolytes such as glycerol possibly induce a quasi-native apo-SaFtsZ state that binds GTP with high affinity (Tables 1, S1, S2).

Folding and binding mechanism. Our solution results can be explained by a simplified model scheme of ligand and osmolyte-induced folding, including transfer equilibria between the aqueous buffer and osmolyte solutions:

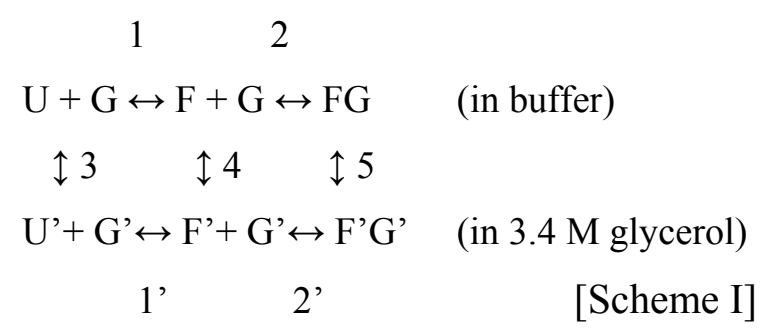

where $U$ represents the unfolded protein, $F$ the folded protein and $G$ guanine nucleotide ligand that is assumed to bind to F only. 
The values of the free energy changes of folding and binding are as follows:

$\Delta \mathrm{G}_{1}=2.6 \pm 0.2 \mathrm{kcal} / \mathrm{mol}$

(in buffer, from two-state model analysis of CD data)

$\Delta \mathrm{G}_{1},=-1.8 \pm 0.3 \mathrm{kcal} / \mathrm{mol}$

(in 3.4 M glycerol, from two-state model analysis of CD data)

$\Delta \mathrm{G}_{2}=-9.2 \pm 0.3 \mathrm{kcal} / \mathrm{mol}$

(average of results of two different measurement procedures)

$\Delta \mathrm{G}_{2},=\Delta \mathrm{G}_{\mathrm{b}, \text { obs } 3.4 \mathrm{M} \mathrm{glyc}}=-10.5 \pm 0.1 \mathrm{kcal} / \mathrm{mol}[\mathbf{5}]$ (ITC results)

The increment free energy changes describing the osmolyte effect on folding and binding equal the increment transfer free energy changes between water and osmolyte solutions in each thermodynamic cycle. They are the coupling free energy changes:

$\Delta \Delta \mathrm{G}$ (folding, transfer $)=\Delta \mathrm{G} 1^{\prime}-\Delta \mathrm{G} 1=\Delta \mathrm{G} 4-\Delta \mathrm{G} 3=-4.4 \pm 0.3 \mathrm{kcal} / \mathrm{mol}$

$\Delta \Delta \mathrm{G}$ (binding, transfer) $=\Delta \mathrm{G} 2^{\prime}-\Delta \mathrm{G} 2=\Delta \mathrm{G} 5-\Delta \mathrm{G} 4=-1.3 \pm 0.2 \mathrm{kcal} / \mathrm{mol}$

Thus the strong effect of $3.4 \mathrm{M}$ glycerol on folding $(-4.4 \mathrm{kcal} / \mathrm{mol})$ equals the more favorable transfer free energy change of the folded than the unfolded protein from water to glycerol solution (equation [6]). This can be interpreted as a more unfavorable repulsion of the osmolyte by unfolded protein than by the folded protein [40]. The dependence of the observed folding equilibrium constant $\left(\mathrm{K}_{\text {fold, obsd }}\right)$ on glycerol activity $\left(\mathrm{a}_{\mathrm{glyc}}\right)$ can be expressed according to linked functions theory [46] as

$\mathrm{d} \ln \left(\mathrm{K}_{\text {fold, obsd }}\right) / \mathrm{d} \ln \left(\mathrm{a}_{\mathrm{glyc}}\right)=\Delta v_{\text {glyc }}$

where $\Delta v_{\text {glyc }}=v_{\text {glyc }}$ (folded) $-v_{\text {glyc }}$ (unfolded) is the change in the number of glycerol molecules thermodynamically bound or repelled by the protein [46]. Notice that from equation [1] it can be deduced that

$\mathrm{d} \ln \left(\mathrm{K}_{\text {fold, obsd }}\right) / \operatorname{dln}\left(\mathrm{a}_{\text {glyc }}\right)=[$ glycerol $] \cdot m / \mathrm{RT}$

and thus, from equations [8] and [9],

$\Delta v_{\mathrm{glyc}}=[$ glycerol $] \cdot \mathrm{m} / \mathrm{RT}$ 
which at 3.4 M glycerol amounts to 7.5 glycerol molecules less that are preferentially excluded by the protein upon folding [36].

The effect of $3.4 \mathrm{M}$ glycerol on binding $(-1.3 \mathrm{kcal} / \mathrm{mol})$ is weaker than on folding. It equals the difference apparent transfer free energy change of the protein-ligand complex minus the separate protein (folded apo-SaFtsZ $\mathrm{T}_{\mathrm{T}}$ ) and ligand (GTP) (equation [7]). Our simplified model includes within the "transfer" free energy change of the folded protein any energetic contribution from small structural rearrangements. The reasonably similar values obtained with the metastable nucleotide-devoid homolog BsFtsZ (-2.0 kcal $/ \mathrm{mol})$ and with the stable apo-MjFtsZ ($0.6 \mathrm{kcal} / \mathrm{mol}$ ) support the model, although minor glycerol-induced folding effects at the NBS cannot be excluded.

The observed enthalpy-driven binding of GTP to apo-SaFtsZ $\mathrm{T}_{\mathrm{T}}$ in $3.4 \mathrm{M}$ glycerol is compatible with a network of hydrogen bonds stabilizing bound nucleotide in FtsZ crystal structures $([7,8,10,20,42]$ and this work). However, GTP binding becomes weakly exothermic and predominantly entropy-driven at low glycerol concentrations (Figure 4E), supporting a positive enthalpy change and a negative entropic contribution for the folding of $\mathrm{SaFts}_{\mathrm{T}}$. These features would be compatible with an increased desolvation of polar and nonpolar groups upon GTP binding to apo-SaFtsZ $\mathrm{T}_{\mathrm{T}}$ [47]. They are in contrast with frequently negative enthalpy and positive entropic contribution to protein folding [48]. The weaker binding of mant-GTP, particularly at high ionic strength, may correspond to a structural rearrangement to accommodate the mant moiety together with the loss of a hydrogen bond from the substituted ribose hydroxyl [43].

Stabilization of folded SaFtsZ by nucleotide and osmolytes. Implications. Stabilization by bound nucleotide may be common among mesophilic FtsZs. For example, the close homolog BsFtsZ has a tendency to sediment upon high-speed centrifugation in the absence of nucleotide, which we suspect may be due to aggregation of a fraction of unfolded apo-protein. The more distantly related Gram-negative EcFtsZ releases nucleotide in low ionic strength buffer [28]. Apo-EcFtsZ is unstable [26] and thus it could not be employed before for biochemical studies. There are also several reports of assembly refractory FtsZs that may be related to nucleotide release [30] [31] [32]. In this work, the reversible unfolding and lack of aggregation of unfolded SaFtsZ have permitted us to elucidate the stabilizing role of nucleotide. Without nucleotide, the normally structured SaFts $Z_{\mathrm{T}}$ core spontaneously unfolds in solution $\left(\Delta \mathrm{G}_{\text {folding }}=+2.6 \mathrm{kcal} \mathrm{mol}^{-1}\right)$. GTP binding (intrinsic $\Delta \mathrm{G}_{\text {binding }}=-9.3 \mathrm{kcal} \mathrm{mol}^{-1}$ ) induces folding of the NBD that remarkably communicates stability to the CTD folded structure. 
FtsZ folding is induced by nucleotide binding

In comparison, eukaryotic tubulin, which shares the FtsZ structural fold, is known to require stabilizing GTP binding during its elaborate chaperone-assisted folding [49], and nucleotide release results in tubulin inactivation [50]. Among classical single domain GTPases, $\mathrm{p} 21^{\mathrm{H}-\mathrm{ras}} 1$ is stabilized by GDP binding, although nucleotide removal leaves most of the secondary structure intact [51], and apo-Rho GTPases remain functional for high affinity nucleotide binding [52].

Interestingly, glycerol and other protein-stabilizing organic osmolytes induce apo$\mathrm{SaFtsZ}_{\mathrm{T}}$ folding and enable high affinity nucleotide binding (Results). These include the bacterial osmolytes trehalose, proline and betaine that replace perturbing inorganic ions in the cytosol to balance osmotic stress [53, 54]. Proline and betaine transport systems enable S. aureus survival in high salt environments, which has been related to infectivity [55]. Increasing ionic strength $(\mathrm{KCl})$ also induces apo-Fts $\mathrm{Z}_{\mathrm{T}}$ folding but strongly reduces the nucleotide binding affinity. It should be noted that 0.5 to $1.5 \mathrm{M} \mathrm{K}^{+}$is physiological for $S$. aureus, which maintains high intracellular $\mathrm{K}^{+}$concentration, even with low extracellular concentration and no osmotic stress [56], while the basal internal $\mathrm{K}^{+}$concentration in B. subtilis is $\sim 0.35 \mathrm{M}$ [54]. Modifying $\mathrm{pH}$ or temperature around physiological values, or addition of macromolecular crowding agents, did not induce high affinity mant-GTP binding (Results). Our in vitro results provide mechanistic insight into the functioning of cell division protein FtsZ. They suggest that upon emerging from the ribosome, SaFtsZ should bind GTP from the cytosol during folding. This would require a free GTP concentration $>>1 \mu \mathrm{M}\left(\mathrm{GTP}_{\mathrm{D}}=1.6 \mu \mathrm{M}\right.$ in $\left.1 \mathrm{M} \mathrm{KCl}\right)$ to effectively bind GTP for folding before assembly. The GTP pool in S. aureus is around $1 \mathrm{mM}$ during growth, which decreases six fold under stringent conditions [57], but the concentration of free GTP/GDP in the cytosol (not bound to the abundant GTP binding proteins) should be significantly less. Thus we cannot discard the possibilities that under starvation (low nucleotide) conditions FtsZ, no longer required for cell division, may adopt a functionally inactive apostructure (suggested by [20] and this work) or unfold and be degraded by proteases including ClpXP $[58,59]$. Nevertheless, it is also possible that osmolytes and ionic strength in bacterial cells protect FtsZ from degradation.

New approaches to FtsZ inhibitor discovery. In addition to our folding and binding studies, glycerol-induced stabilization of apo-SaFts $Z_{\mathrm{T}}$ has permitted the formulation of this protein in a competition assay designed to detect any molecules that may bind overlapping the GTP site and determine their apparent affinity. In practice, $25 \%(\mathrm{w} / \mathrm{v})$ glycerol may be replaced if required by other osmolytes, including $25 \%$ (w/v) ethyleneglycol, $1 \mathrm{M}$ TMAO, betaine, trehalose or possibly 
$0.25 \mathrm{M}$ KAcetate. The robust fluorescence anisotropy competitive assay developed has good sensitivity, uses low concentrations of protein and probe, and may be easily adapted to microwell plate readers. It provides a solid starting point to robotic high throughput screening for high affinity inhibitors targeting the GTP binding site of Gram-positive S. aureus FtsZ. Fluorescence and ITC binding measurements should be combined with phenotypically characterizing inhibitor effects on bacterial division [60]. On the other hand, very high-affinity ligands, binding to the GTP or other site, may induce apo-SaFtsZ $\mathrm{T}_{\mathrm{T}}$ folding in the absence of osmolytes, so that they could be picked up by an appropriate fluorescence thermal shift screen designed to detect apo$\mathrm{SaFtsZ}_{\mathrm{T}}$ stabilization. It may be hypothesized that certain GTP-replacing inhibitors could impair the correct folding of the NBS, or of the NBD and CTD of FtsZ, thus poisoning its assembly function. We have additionally prepared EcFtsZ predominantly devoid of nucleotide and employed the fluorescence anisotropy competition assay to show the feasibility of screening as well for inhibitors of FtsZ from Gram-negative pathogens. Given the stabilizing effect of bound nucleotide and the differences observed in between different nucleotide-devoid FtsZs, their effective affinities for GTP-replacing inhibitors may be different, in spite of the conservation of the NBS. This suggests the possibility of selectively targeting FtsZ on pathogenic bacteria with GTP-replacing inhibitors not harmful to the gut microbiota.

By examining the crystal structures of GDP-bound SaFts $\mathrm{Z}_{\mathrm{T}}$ in the absence and presence of small compounds, we identified a cavity that is able to harbor different small ligands, such as 1-ethylene glycol, methyl-2-pyrrolidone, and 2-methyl-2,4-pentanediol. This promiscuous cavity locates at the deepest part of the NBS, in close proximity to the guanine moiety of GDP, and it is expected to be amenable to crystallographic fragment screening. In this sense, several C8susbtituted guanine nucleotide inhibitors, including 8-pyrrolidino-GTP, were predicted to enter this cavity from NMR and modeling results [61]. Therefore, it can be envisioned that chemical compounds that extend the GDP molecule to fill this cavity will likely displace the natural nucleotides from their binding pocket and thus inhibit FtsZ function. Exploring a diverse set of compounds with increased affinity would open the way for the design of new nucleotidereplacing inhibitors with potential therapeutic significance.

\section{Methods}

Protein purification. Untagged full length FtsZ from S. aureus $\left(\mathrm{SaFts}_{\mathrm{wt}}\right)$ was purified as described [19] except for the gel-filtration chromatography which was run in a Superdex 75 column (XK 1.6/70 cm, GE Healthcare) with $50 \mathrm{mM}$ Tris- $\mathrm{HCl}, 50 \mathrm{mM} \mathrm{KCl}, 1 \mathrm{mM}$ EDTA, $10 \%$ 
glycerol, pH 7.5, with $100 \mu \mathrm{M}$ GDP (sodium salt, Sigma) at $4{ }^{\circ} \mathrm{C} .2 \mathrm{mM}$ GDP was added to the protein before loading into the column. The N-terminal sequence of $\mathrm{SaFts}_{\mathrm{wt}}$ was confirmed by Edman microsequencing with an Applied Biosystems Procise 494 sequencer. SaFts $Z_{\mathrm{wt}}$ preparations had $0.89 \pm 0.09$ guanine nucleotide bound per FtsZ.

His-tagged full length FtsZ from $S$. aureus $\left(\mathrm{SaFtsZ}_{\mathrm{f}}\right)$ was overexpressed in E. coli BL21 (DE3) (2 L culture) transformed with a pET28a plasmid in which a 1188 bp fragment containing the S. aureus (Mu50/ATCC 700699) ftsZ ORF was cloned. SaftsZ was amplified using primers SaFtsZpET28-F (5'- TTTCATATGTTAGAATTTGAACAAGGA-3') and SaFtsZpET28-R (5'AAAGGATCCTTAACGTCTTGTTCTTCT-3'). The PCR product was digested with NdeI and BamHI (sites underlined in primer sequences above) and cloned into pET28a, cut with the same enzymes. SaFts $Z_{F}$ was affinity purified using HiTrap Chelating columns (5 mL, GE Healthcare) in $50 \mathrm{mM}$ Tris- $\mathrm{HCl}, 50 \mathrm{mM} \mathrm{KCl}, 10 \%$ glycerol, $\mathrm{pH} 8.0$ with an imidazole gradient $(50 \mathrm{mM}$ to 1 $\mathrm{M})$. The fractions were analyzed by SDS-PAGE and those containing the protein were pooled and diluted to $40 \mathrm{~mL}$ with ion-exchange buffer A. The His-tag was cleaved overnight at $4^{\circ} \mathrm{C}$ with bovine plasma thrombin (100 NIH units; Sigma), yielding a protein with 3 extra N-terminal residues GSH-SaFtsZ(1-390). The protein (diluted to $100 \mathrm{~mL}$ with ion-exchange buffer $\mathrm{A}$ and adjusted to $5 \mathrm{mM} \mathrm{MgCl}_{2}$ ) was loaded into a HiTrap Chelating column (to retain any uncleaved His-tagged protein) connected in tandem with an anion exchange HiTrapQ HP column (5 mL, GE Healthcare) in $50 \mathrm{mM}$ Tris- $\mathrm{HCl}, \mathrm{pH} 7.5,5 \mathrm{mM} \mathrm{MgCl}_{2}, 10 \%$ glycerol, that was eluted with a $\mathrm{KCl}$ gradient (buffer $\mathrm{A}: 50 \mathrm{mM}$ to buffer $\mathrm{B}$ : $1 \mathrm{M}$ ). The protein was concentrated to $2 \mathrm{~mL}$ and Superdex 75 size exclusion chromatography was performed as above. The $\mathrm{N}$-terminal sequence of SaFtsZ $Z_{f}$ was confirmed by Edman microsequencing. SaFts $Z_{f}$ contained $0.77 \pm 0.07$ guanine nucleotide bound per monomer.

Truncated FtsZ from S. aureus containing residues 12-316 $\left(\mathrm{SaFts}_{\mathrm{T}}\right)$ was overexpressed in E. coli BL21 (DE3) (2 L culture) transformed with a pET28a plasmid in which a $936 \mathrm{bp}$ fragment containing the SaftsZ ORF from nucleotide 33 to nucleotide 945 was cloned. The fragment was amplified using primers SaFtsZ12-NdeI (5'TTTCATATGGCGACTTTAAAGGTCAT-3') and SaFtsZ316-BamHI (5'AAAGGATCCTCAATCAAAACCAGTTG-3'). The PCR product was digested with NdeI and BamHI (sites underlined in primer sequences above) and cloned into pET28a. SaFtsZ $\mathrm{T}_{\mathrm{T}}$ was purified as described for $\mathrm{SaFts}_{\mathrm{f}}$ above with minor modifications, yielding a construct with 4 extra N-terminal residues GSHM-SaFtsZ(12-316) after thrombin removal of the His tag. The buffer employed in the Superdex 75 size exclusion chromatography was $10 \mathrm{mM}$ Tris- $\mathrm{HCl}, 50$ 
FtsZ folding is induced by nucleotide binding $\mathrm{mM} \mathrm{KCl}, \mathrm{pH}$ 7.5, $100 \mu \mathrm{M}$ GDP (without glycerol). SaFtsZ $\mathrm{T}_{\mathrm{T}}$ eluted at a volume Ve/Vo = 1.44, corresponding to the size of the construct. The molecular weight of $\mathrm{SaFts}_{\mathrm{T}}$ was confirmed by mass spectroscopy at the CIB Proteomics Facility with a MALDI-TOF-TOF Autoflex III (Bruker Daltonics, Bremen, Germany) (31910 Da, theor. 31914 Da). SaFtsZ $\mathrm{T}_{\mathrm{T}}$ contained $0.78 \pm$ 0.07 guanine nucleotide bound. The typical yield was $30-40 \mathrm{mg}$ protein per $2 \mathrm{~L}$ culture.

To obtain nucleotide-devoid truncated FtsZ from S. aureus (apo-SaFtsZ $Z_{\mathrm{T}}$ ), GSHMSaFtsZ(12-316), the SaFtsZ $\mathrm{T}_{\mathrm{T}}$ procedure was modified. After loading the protein into the Niaffinity HiTrap Chelating column, it was washed with 50 column volumes of loading buffer to facilitate nucleotide release. The fractions from anion exchange chromatography containing nucleotide-devoid protein were selected by measuring their UV absorption spectra (minimum absorbance ratio $\mathrm{A}_{254} \mathrm{~nm} / \mathrm{A}_{280} \mathrm{~nm}$ ). The Superdex 75 size exclusion chromatography was performed in $10 \mathrm{mM}$ Tris- $\mathrm{HCl}, 50 \mathrm{mM} \mathrm{KCl}, \mathrm{pH} 7.5$ without glycerol or nucleotide. The nucleotide-devoid protein eluted near the exclusion volume, $\mathrm{Ve} / \mathrm{Vo}=1.14$, indicating a considerable larger hydrodynamic size than expected from a globular 32000 Da monomer. Apo$\mathrm{SaFts}_{\mathrm{T}}$ contained undetectable guanine nucleotide. The typical yield was 20-25 mg protein per 2L culture.

SaFtsZ concentration was measured with the BioRad Bradford protein assay using spectrophotometrically calibrated BsFtsZ standards, due to the lack of Tyr and Trp residues. The nucleotide content of the protein was determined spectrophotometrically after perchloric acid extraction [62]. BsFtsZ, full length untagged FtsZ from B. subtilis, was purified with $\sim 0.05$ nucleotide bound [18]. Nucleotide-devoid FtsZ from M. jannaschii (apo-MjFtsZ) was prepared as described [26]. Full length FtsZ from E. coli (EcFtsZ) was purified with $\sim 0.8$ guanine nucleotide bound as described [62]. These proteins were stored at $-75^{\circ} \mathrm{C}$.

Nucleotide-devoid EcFtsZ was prepared by incubating EcFtsZ $(\sim 0.2 \mathrm{mM})$ for $10 \mathrm{~min}$ at room temperature in low ionic strength buffer $10 \mathrm{mM}$ Tris, 1mM EDTA, pH 7.5 (0.8 mL), followed by gel filtration on a 0.9 x $25 \mathrm{~cm}$ Sephadex G-25 column, slowly run at $0.27 \mathrm{ml} / \mathrm{min}$ in the same buffer, to separate the protein from the released nucleotide. As the $1 \mathrm{ml}$ protein fractions left the column, they were diluted with one volume of $50 \mathrm{mM}$ Pipes-KOH, $500 \mathrm{mM}$ $\mathrm{KCl}, 1 \mathrm{mM}$ EDTA, pH 7.5, 3.4 M glycerol. This apo-EcFtsZ contained residual 0.3 guanine nucleotide bound per FtsZ and assembled similarly to EcFtsZ in light scattering tests. It was possible to reduce the nucleotide content of the protein to $\sim 0.1$ by reducing the flow of the gel filtration column to $0.16 \mathrm{ml} / \mathrm{min}$, but a reduced mant-GTP anisotropy and assembly activity were observed. Apo-EcFtsZ was stable for several days at $4^{\circ} \mathrm{C}$. 
Circular dichroism and additive-induced folding. CD spectra were acquired with a Jasco J720-715 spectropolarimeter employing 0.2-mm cells in a thermostatted cell holder. Four scans of each protein sample ( $1 \mathrm{~nm}$ bandwidth measurement interval, $20 \mathrm{~nm} \mathrm{~min}{ }^{-1}$ scan speed and $4 \mathrm{~s}$ time constant) were averaged but not smoothed and solvent baseline was subtracted. Mean residue mass ellipticities were calculated based on the average molecular mass per residue obtained from the amino acid composition. Spectra were acquired in $10 \mathrm{mM}$ Tris-HCl, $50 \mathrm{mM}$ $\mathrm{KCl}$, pH 7.5 at $25{ }^{\circ} \mathrm{C}$, unless indicated. Additives employed were: glycerol and ethyleneglycol (Merck, for analysis), polyethyleneglycol 5000 monomethyl ether (PEG5000 MME employed for crystallization; Aldrich), Ficoll PM70 (Amersham Biosciences), trimethylamine-N-oxide dihydrate (TMAO, $\geq 99 \%$, Sigma-Aldrich; found free from UV absorbing fluorescent contaminants), 2-methyl-2,4-pentanediol (MPD, 99\%, Merck), D(+)-trehalose dihydrate (VWR chemicals), betaine ( $>98 \%$, Sigma-Aldrich), L-proline (99\%, Sigma-Aldrich), guanidinium chloride (99.9\%, Calbiochem), dimethylsulfoxide (DMSO, Merck, for spectroscopy). For the analysis of additive-induced $\mathrm{SaFtsZ}_{\mathrm{T}}$ folding experiments the ellipticity at $222 \mathrm{~nm}(\theta)$ as a function of the stabilizer concentration in molar units $([\mathrm{X}])$, the expression $\theta=\left(\theta_{\min }+\left(\Delta \theta_{\max }+\right.\right.$ $s[\mathrm{X}]) \cdot \exp (-\Delta \mathrm{G} / 0.5925+m \cdot[\mathrm{X}] / \mathrm{RT})) /(1+\exp (-\Delta \mathrm{G} / \mathrm{RT}+m \cdot[\mathrm{X}] / \mathrm{RT}))$ was fit to the data, where $\Delta \mathrm{G}$ is the folding free energy change $\left(\mathrm{cal} \cdot \mathrm{mol}^{-1}\right), m$ the efficiency parameter of the stabilizer and $s$ in the slope of the postfolding region (derived from the equation for unfolding [38, 39].

NMR spectroscopy. NMR spectra were recorded on a Bruker $600 \mathrm{MHz}\left({ }^{1} \mathrm{H}\right)$ instrument equipped with a cryoprobe and Z-gradients. Spectra were registered at $25.0^{\circ} \mathrm{C}$ on SaFtsZ samples after transfer to buffer containing $10 \mathrm{mM}$ deuterated Tris (D11, 98\%, Cambridge Isotope Labs), $50 \mathrm{mM} \mathrm{KCl}\left(\mathrm{pH}^{*} 7.4\right)$ and $100 \% \mathrm{D}_{2} \mathrm{O}$, using a $\mathrm{HR} 10 / 10$ fast desalting column (Pharmacia Biotech). $\mathrm{pH}^{*}$ is the $\mathrm{pH}$ meter reading in $100 \% \mathrm{D}_{2} \mathrm{O}$ without correction for the deuterium isotope effect. Sodium 4,4-dimethyl-4-silapentane-1-sulfonate (DSS) added to a final concentration of $0.20 \mathrm{mM}$ as the chemical shift reference. A series of $1 \mathrm{D}{ }^{1} \mathrm{H}$ NMR spectra (acquisition time $=5.33$ minutes) were recorded on apo-SaFts $Z_{\mathrm{T}}$ before and following the addition of GTP to a final concentration of $2.0 \mathrm{mM}$. Spectra were transformed and analyzed using the Bruker Topspin program, version 4.0.2. To evaluate the folding kinetics, a spectral zone harboring chiefly denatured signals; namely: 1.02 to $0.80 \mathrm{ppm}$ as well as spectral regions containing native peaks; namely 0.68 to $0.52 \mathrm{ppm}, 0.51$ to $0.38 \mathrm{ppm}$, and -0.03 to $-0.17 \mathrm{ppm}$ were integrated. The peak of the chemical shift reference, DSS at +0.03 to $-0.03 \mathrm{ppm}$ was also integrated and used an intensity standard for the other peaks. A single exponential equation: $A_{t}$ $=A_{0} \cdot e^{-k t}+A_{\infty}$, where $A_{t}, A_{0}$ and $A_{\infty}$ are the integrals at times $t, 0$, and infinity, respectively, $k$ is 
the observed rate constant and $\mathrm{t}$ is time in minutes, was fit to the signal area versus time data using the program Kaleidagraph.

Analysis of FtsZ polymerization. SaFtsZ assembly experiments were performed in $50 \mathrm{mM}$ Mes, $50 \mathrm{mM} \mathrm{KCl}, 1 \mathrm{mM}$ EDTA, $10 \mathrm{mM} \mathrm{MgCl}_{2}$, pH 6.5, at $25^{\circ} \mathrm{C}$. Polymerization was started by addition of $0.05 \mathrm{mM}$ GMPCPP (Guanosine-5'-[( $\alpha, \beta)$-methyleno]triphosphate, sodium salt, Jena Bioscience) or $1 \mathrm{mM}$ GTP (lithium salt, Sigma) and it was monitored by right angle light scattering at $350 \mathrm{~nm}(0.5 \mathrm{~nm}$ excitation and emission band-pass $)$ using a Fluromax-4 spectrofluorometer. Aliquots of the polymer solutions were adsorbed to carbon-coated grids, negatively strained with 2\% uranyl acetate and examined with a Jeol 1230 electron microscope operated at $100 \mathrm{kV}$. Sa-FtsZ assembly was also analyzed by polymer sedimentation; after nucleotide addition, samples were centrifuged at $386000 \mathrm{~g}$ for $20 \mathrm{~min}$ in a Beckman TLA100 rotor in a TLX ultracentrifuge. The supernatants were withdrawn carefully and the pellets resuspended in the same volume of buffer. Pellets and their corresponding supernatants were loaded and run in the same well of an SDS-PAGE $12 \%$ gel with a 20 min delay. Gels were stained with Coomasie blue, scanned with a CS-800 calibrated densitometer (BioRad) and analyzed with Quantity One software (BioRad) to measure the amount of protein polymerized.

Analytical ultracentrifugation. Sedimentation velocity experiments were carried out with a Beckman Optima XL-1 analytical ultracentrifuge equipped with interference optics, using an An50/Ti rotor with 12-mm double-sector centerpieces at $48000 \mathrm{rpm}$, and at $25{ }^{\circ} \mathrm{C}$, in $10 \mathrm{mM}$ Tris-HCl, $50 \mathrm{mM} \mathrm{KCl,} \mathrm{pH} \mathrm{7.5.} \mathrm{Differential} \mathrm{sedimentation} \mathrm{coefficients} \mathrm{distributions,} \mathrm{c(s),} \mathrm{were}$ calculated with SEDFIT [63]. The weight average sedimentation coefficient values measured in buffer at $25^{\circ} \mathrm{C}$ were corrected to $\mathrm{H}_{2} \mathrm{O}$ at $20^{\circ} \mathrm{C}$, s $20, w$.

Isothermal Titration Calorimetry. ITC measurements [64] were performed using a MicroCal PEAQ-ITC calorimeter (Malvern) at $25^{\circ} \mathrm{C}$. The sample cell $(0.2 \mathrm{~mL})$ was loaded with $20-25 \mu \mathrm{M}$ FtsZ carefully diluted in buffer and the syringe was loaded with the nucleotides diluted in exactly the same buffer to final concentration comprised between 200 and $700 \mu \mathrm{M}$. Buffer was $25 \mathrm{mM}$ Pipes-KOH, $50 \mathrm{mM} \mathrm{KCl,} 1 \mathrm{mM}$ EDTA, glycerol, $\mathrm{pH}$ 7.5. Protein samples were always incubated for $30 \mathrm{~min}$ in buffer with glycerol or $\mathrm{KCl}$ to allow folding. All samples were degassed before loading. As a control experiment, the individual dilution heats for the syringe reactant were determined by carrying out identical injections of the reactant into the sample cell loaded with buffer. Controls without nucleotide and buffer in buffer controls were also run. The electrical power required to maintain the reaction cell at constant temperature after each injection was recorded as a function of time, generating the thermograms shown. Binding isotherms were 
FtsZ folding is induced by nucleotide binding fit to a single type of site model, correcting for dilution effects, using the MicroCal PEAQ-ITC Analysis software.

Crystallization and structure determination. Crystals were grown by vapor diffusion at 295 $\mathrm{K}$, mixing $1 \mu \mathrm{L}$ of the protein with $1 \mu \mathrm{L}$ of the crystallization solution around previously reported conditions [20]. Well-diffracting, plate-shaped crystals typically appeared after several days in $0.2 \mathrm{M}$ lithium sulfate, $10 \%$ ethyleneglycol, $0.1 \mathrm{M}$ Tris- $\mathrm{HCl} \mathrm{pH}$ 8.4-9.0, 24-28\% PEG5000 MME. Refolded apo-SaFtsZ $(25 \mathrm{mg} / \mathrm{mL})$ was in $10 \mathrm{mM}$ Tris-HCl pH 7.5, $250 \mathrm{mM}$ $\mathrm{mM}$ KAcetate, $800 \mu \mathrm{M}$ GDP. SaFtsZ $\mathrm{T}_{\mathrm{T}}$ GDP $(14 \mathrm{mg} / \mathrm{mL})$ was in $10 \mathrm{mM}$ Tris- $\mathrm{HCl} \mathrm{pH}$ 7.5, 300 $\mathrm{mM} \mathrm{KCl}, 100 \mu \mathrm{M}$ GDP. For the SaFtsZ $\mathrm{T}_{\mathrm{T}}$-MetPyr structure, the protein solution contained 4\% (v/v) 1-methyl-2-pyrrolidone (Sigma, analytical standard) and the crystals were soaked in 10\% $(\mathrm{v} / \mathrm{v})$ of this co-solvent in reservoir solution. SaFts $\mathrm{Z}_{\mathrm{T}}$-GDP crystals were harvested in cryo-loops and plunged into liquid nitrogen without further cryoprotection. SaFts $\mathrm{Z}_{\mathrm{T}}$-Apo crystals without bound nucleotide were grown by mixing $0.2 \mu \mathrm{L}$ of SaFtsZ $\mathrm{T}_{\mathrm{T}} \mathrm{GDP}$ with $0.2 \mu \mathrm{L}$ of a solution containing 2.1 M ammonium sulfate, $0.1 \mathrm{Mes}-\mathrm{NaOH} \mathrm{pH}$ 6.2, and were cryoprotected with $20 \%$ glycerol.

All X-ray diffraction data were collected at $100 \mathrm{~K}$ on beamline Xaloc at the ALBA synchrotron (Spain) and processed using XDS [65] and programs of the CCP4 Suite [66]. The structures were determined through molecular replacement using Phaser [67], with PDB entry 3VOA for SaFtsZ-refold and SaFtsZ-GDP, and 3VPA for SaFtsZ-Apo. The atomic models were rebuilt using Coot [68] and refined with PHENIX [69]. Data collection and refinement statistics are summarized in Table S1. All figures were prepared using Pymol (Schrödinger Inc.). The coordinates and structure factors of refolded $\mathrm{SaFts}_{\mathrm{T}}, \mathrm{SaFts}_{\mathrm{T}}-\mathrm{Apo}, \mathrm{SaFts}_{\mathrm{T}}-\mathrm{GDP}-\mathrm{Wat}$, SaFtsZ $_{\mathrm{T}}$-GDP-MetPyr, SaFtsZ $\mathrm{T}_{\mathrm{T}}$-GDP-EthGly have been deposited in the RCSB Protein Data Bank under accession codes 6SI9, 6RVM, 6RVN, 6RVP, 6RVQ, respectively.

Mant-GTP binding to FtsZ and displacement by inhibitors. The equilibrium binding constant of mant-GTP (2'/3'-O-(N-Methyl-anthraniloyl)-guanosine-5'-triphosphate, triethylammonium salt, Jena Bioscience) was measured through changes in its fluorescence anisotropy upon binding to apo-SaFtsZ ${ }_{\mathrm{T}}\left(\mathrm{r}_{\text {free }}=0.021 \pm 0.006 ; \mathrm{r}_{\text {bound }}=0.223 \pm 0.005\right.$, correcting for the 2.8 -fold intensity change between free and bound ligand; Fig. S9). These experiments and ligand competition with mant-GTP were performed as previously described in detail for MjFtsZ [27] and BsFtsZ [18]. Here, protein samples were incubated in buffer with glycerol or the different additives and mantGTP for $30 \mathrm{~min}$, then the competing ligand was added to aliquots of the solution and fluorescence anisotropy was measured $10 \mathrm{~min}$ later. Conditions: $10 \mathrm{mM}$ Tris- $\mathrm{HCl}, 50 \mathrm{mM} \mathrm{KCl}$, 
FtsZ folding is induced by nucleotide binding $3.4 \mathrm{M}$ glycerol, $\mathrm{pH} 7.5,2 \%(\mathrm{v} / \mathrm{v}) \mathrm{DMSO}, 25{ }^{\circ} \mathrm{C}$. All measurements were acquired with a Fluoromax-4 (Horiba Jobin Yvon) photon-counting spectrofluorometer, with excitation at 357 (5 $\mathrm{nm}$ band pass) and emission at $445 \mathrm{~nm}$ (10 nm band pass) using $2 \times 10 \mathrm{~mm}$ cells at $25^{\circ} \mathrm{C}$. Binding of mant-GTP by excess apo-EcFtsZ resulted in a maximal anisotropy $\mathrm{r}_{\text {bound }}=0.216$; $(2.6$-fold intensity change between free and bound mant-GTP). Competition of GDP and the synthetic ligands (UCM44 and UCM81) with mant-GTP were similarly performed.

Author contributions: SH, AJCV, FMR, DL and CFT planned and performed experiments and analysed data, LAB contributed essential materials, DL, CFT and JMA conceived the study, analysed data and wrote the paper.

Acknowledgments: We thank Margarita Menéndez (IQFR) for extensive thermodynamic discussions and equation [10], Antonio Romero and F. Javier Medrano (CIB) for support with Xray diffraction, and David Juan (CIB) for protein purification. We are grateful to the ALBA staff for helpful assistance.

This work was supported by grants BFU 2014-51823-R (JMA), BFU 2017-87387-P (CFT), SAF-2016-76678-C2-2-R (DVL) and a FPI fellowship (AJCV). NMR experiments were performed in the "Manuel Rico" NMR laboratory (LMR) of the Spanish National Research Council (CSIC), a node of the Spanish Large-Scale National Facility (ICTS R-LRB).

JMA wishes to dedicate this study to the memory of Professor Serge N. Timasheff (19262019) for his inspiring work [36, 70], mentoring and long-time friendship.

\section{References}

1. Bi, E. \& Lutkenhaus, J. (1991) FtsZ ring structure associated with division in Escherichia-coli, Nature. 354, 161-164.

2. den Blaauwen, T., Hamoen, L. \& Levin, P. A. (2017) The divisome at 25: the road ahead, Current Opinion in Microbiology. 36, 85-94.

3. Bisson-Filho, A. W., Hsu, Y. P., Squyres, G., Kuru, E., Wu, F., Jukes, C., Dekker, C., Holden, S. J., VanNieuwenhze, M., Brun, Y. V. \& Garner, E. (2017) Treadmilling by FtsZ filaments drives peptidoglycan synthesis and bacterial cell division, Science. 355, 739-743.

4. Yang, X., Lyu, Z., Miguel, A., McQuillen, R., Huang, K. C. \& Xiao, J. (2017) GTPase activity-coupled treadmilling of the bacterial tubulin FtsZ organizes septal cell-wall synthesis, Science. 355, 744-747.

5. Monteiro, J. M., Pereira, A. R., Reichmann, N. T., Saraival, B. M., Fernandes, P. B., Veiga, H., Tavares, A. C., Santos, M., Ferreira, M. T., Macario, V., VanNieuwenhze, M. S., Filipe, S. R. \& Pinho, M. G. (2018) Peptidoglycan synthesis drives an FtsZ-treadmilling-independent step of cytokinesis, Nature. 554, 528532.

6. Perez, A. J., Cesbron, Y., Shaw, S. L., Bazan Villicana, J., Tsui, H.-C. T., Boersma, M. J., Ye, Z. A., Tovpeko, Y., Dekker, C., Holden, S. \& Winkler, M. E. (2019) Movement dynamics of divisome proteins and PBP2x:FtsW in cells of Streptococcus pneumoniae, Proceedings of the National Academy of Sciences of the United States of America. 116, 3211-3220.

7. Nogales, E., Downing, K. H., Amos, L. A. \& Lowe, J. (1998) Tubulin and FtsZ form a distinct family of GTPases, Nature Structural Biology. 5, 451-458. 
8. Elsen, N. L., Lu, J., Parthasarathy, G., Reid, J. C., Sharma, S., Soisson, S. M. \& Lumb, K. J. (2012) Mechanism of Action of the Cell-Division Inhibitor PC190723: Modulation of FtsZ Assembly Cooperativity, Journal of the American Chemical Society. 134, 12342-12345.

9. Artola, M., Ruiz-Avila, L. B., Ramirez-Aportela, E., Martinez, R. F., Araujo-Bazán, L., Vázquez-Villa, H., Martín-Fontecha, M., Oliva, M. A., Martín-Galiano, A. J., Chacón, P., López-Rodríguez, M. L., Andreu, J. M. \& Huecas, S. (2017) The structural assembly switch of cell division protein FtsZ probed with fluorescent allosteric inhibitors, Chemical Science. 8, 1525-1534.

10. Wagstaff, J. M., Tsim, M., Oliva, M. A., García-Sanchez, A., Kureisaite-Ciziene, D., Andreu, J. M. \& Löwe, J. (2017) A polymerisation-associated conformational switch in FtsZ, mBio. 8, e00254-17.

11. Buske, P. J., Mittal, A., Pappu, R. V. \& Levin, P. A. (2015) An intrinsically disordered linker plays a critical role in bacterial cell division, Seminars in Cell \& Developmental Biology. 37, 3-10.

12. Sundararajan, K. \& Goley, E. D. (2017) The intrinsically disordered C-terminal linker of FtsZ regulates protofilament dynamics and superstructure in vitro, Journal of Biological Chemistry. 292, 20509-20527.

13. Huecas, S., Ramirez-Aportela, E., Vergonos, A., Nunez-Ramirez, R., Llorca, O., Fernando Diaz, J., JuanRodriguez, D., Oliva, M. A., Castellen, P. \& Andreu, J. M. (2017) Self-Organization of FtsZ Polymers in Solution Reveals Spacer Role of the Disordered C-Terminal Tail, Biophysical Journal. 113, 1831-1844.

14. Haydon, D. J., Stokes, N. R., Ure, R., Galbraith, G., Bennett, J. M., Brown, D. R., Baker, P. J., Barynin, V. V., Rice, D. W., Sedelnikova, S. E., Heal, J. R., Sheridan, J. M., Aiwale, S. T., Chauhan, P. K., Srivastava, A., Taneja, A., Collins, I., Errington, J. \& Czaplewski, L. G. (2008) An inhibitor of FtsZ with potent and selective anti-staphylococcal activity, Science. 321, 1673-1675.

15. Ventola, C. L. (2015) The antibiotic resistance crisis: part 1: causes and threats, Pharmacy and Therapeutics : a peer-reviewed journal for formulary management. 40, 277-83.

16. Foster, T. J. (2019) Can beta-Lactam Antibiotics Be Resurrected to Combat MRSA?, Trends in Microbiology. 27, 26-38.

17. Läppchen, T., Pinas, V. A., Hartog, A. F., Koomen, G. J., Schaffner-Barbero, C., Andreu, J. M., Trambaiolo, D., Lowe, J., Juhem, A., Popov, A. V. \& den Blaauwen, T. (2008) Probing FtsZ and tubulin with C8-substituted GTP analogs reveals differences in their nucleotide binding sites, Chem Biol. 15, 18999.

18. Ruiz-Avila, L. B., Huecas, S., Artola, M., Vergonos, A., Ramirez-Aportela, E., Cercenado, E., Barasoain, I., Vazquez-Villa, H., Martin-Fontecha, M., Chacon, P., Lopez-Rodriguez, M. L. \& Andreu, J. M. (2013) Synthetic Inhibitors of Bacterial Cell Division Targeting the GTP-Binding Site of FtsZ, ACS Chem Biol. 8, 2072-2083.

19. Andreu, J. M., Schaffner-Barbero, C., Huecas, S., Alonso, D., Lopez-Rodriguez, M. L., Ruiz-Avila, L. B., Nunez-Ramirez, R., Llorca, O. \& Martin-Galiano, A. J. (2010) The antibacterial cell division inhibitor PC190723 is a FtsZ polymer stabilizing agent which induces filament assembly and condensation, $J$ Biol Chem, 285,14239-14246.

20. Matsui, T., Yamane, J., Mogi, N., Yamaguchi, H., Takemoto, H., Yao, M. \& Tanaka, I. (2012) Structural reorganization of the bacterial cell-division protein FtsZ from Staphylococcus aureus, Acta Cryst Sect DBiol Crystallography. 68, 1175-1188.

21. Tan, C. M., Therien, A. G., Lu, J., Lee, S. H., Caron, A., Gill, C. J., Lebeau-Jacob, C., Benton-Perdomo, L., Monteiro, J. M., Pereira, P. M., Elsen, N. L., Wu, J., Deschamps, K., Petcu, M., Wong, S., Daigneault, E., Kramer, S., Liang, L. Z., Maxwell, E., Claveau, D., Vaillancourt, J., Skorey, K., Tam, J., Wang, H., Meredith, T. C., Sillaots, S., Wang-Jarantow, L., Ramtohul, Y., Langlois, E., Landry, F., Reid, J. C., Parthasarathy, G., Sharma, S., Baryshnikova, A., Lumb, K. J., Pinho, M. G., Soisson, S. M. \& Roemer, T. (2012) Restoring Methicillin-Resistant Staphylococcus aureus Susceptibility to beta-Lactam Antibiotics, Science Transl Med. 4, 126ra35.

22. Fujita, J., Maeda, Y., Mizohata, E., Inoue, T., Kaul, M., Parhi, A. K., LaVoie, E. J., Pilch, D. S. \& Matsumura, H. (2017) Structural Flexibility of an Inhibitor Overcomes Drug Resistance Mutations in Staphylococcus aureus FtsZ, Acs Chemical Biology. 12, 1947-1955.

23. Plaza, A., Keffer, J. L., Bifulco, G., Lloyd, J. R. \& Bewley, C. A. (2010) Chrysophaentins A-H, Antibacterial Bisdiarylbutene Macrocycles That Inhibit the Bacterial Cell Division Protein FtsZ, Journal of the American Chemical Society. 132, 9069-9077. 
24. Artola M., Ruiz-Avila L.B., Vergoñós A., Huecas S., Araujo-Bazán L., Martín-Fontecha M., VázquezVilla H., Turrado C., Ramírez-Aportela E., Hoegl A., Nodwell A., Barasoain I., Chacón P., Sieber S.A., Andreu J.M. \& M., L.-R. (2015) Effective GTP-replacing FtsZ Inhibitors and Antibacterial Mechanism of Action, ACS Chem Biol. 10, 834-843.

25. Steinmetz, M. O. \& Prota, A. E. (2018) Microtubule-Targeting Agents: Strategies To Hijack the Cytoskeleton, Trends in Cell Biology. 28, 776-792.

26. Huecas, S. \& Andreu, J. M. (2004) Polymerization of nucleotide-free, GDP- and GTP-bound cell division protein FtsZ: GDP makes the difference, Febs Letters. 569, 43-48.

27. Schaffner-Barbero, C., Gil-Redondo, R., Ruiz-Avila, L. B., Huecas, S., Lappchen, T., den Blaauwen, T., Diaz, J. F., Morreale, A. \& Andreu, J. M. (2010) Insights into nucleotide recognition by cell division protein FtsZ from a mant-GTP competition assay and molecular dynamics, Biochemistry. 49, 10458-72.

28. Andreu, J. M., Oliva, M. A. \& Monasterio, O. (2002) Reversible unfolding of FtsZ cell division proteins from archaea and bacteria, Journal of Biological Chemistry. 277, 43262-43270.

29. Montecinos-Franjola, F., Ross, J. A., Sanchez, S. A., Brunet, J. E., Lagos, R., Jameson, D. M. \& Monasterio, O. (2012) Studies on the Dissociation and Urea-Induced Unfolding of FtsZ Support the Dimer Nucleus Polymerization Mechanism, Biophysical Journal. 102, 2176-2185.

30. Mukherjee, A. \& Lutkenhaus, J. (1998) Dynamic assembly of FtsZ regulated by GTP hydrolysis, Embo Journal. 17, 462-469.

31. Chen, Y., Anderson, D. E., Rajagopalan, M. \& Erickson, H. P. (2007) Assembly dynamics of mycobacterium tuberculosis FtsZ, Journal of Biological Chemistry. 282, 27736-27743.

32. Milam, S. L. \& Erickson, H. P. (2013) Rapid in Vitro Assembly of Caulobacter crescentus FtsZ Protein at pH 6.5 and 7.2, Journal of Biological Chemistry. 288, 23675-23679.

33. Greenfield, N. J. (2006) Using circular dichroism spectra to estimate protein secondary structure, Nature Protocols. 1, 2876-2890.

34. Bai, Y. W., Milne, J. S., Mayne, L. \& Englander, S. W. (1993) Primary structure effects on peptide group hydrogen-exchange, Proteins-Structure Function and Genetics. 17, 75-86.

35. Bundi, A. \& Wuthrich, K. (1979) H-1-NMR parameters of the common amino-acid residues measured in aqueous solutions of the linear tetrapeptides H-Gly-Gly-X-L-Ala-OH, Biopolymers. 18, 285-297.

36. Gekko, K. \& Timasheff, S. N. (1981) Mechanism of protein stabilization by glycerol - Preferential hydration in glycerol-water mixtures, Biochemistry. 20, 4667-4676.

37. Vagenende, V., Yap, M. G. S. \& Trout, B. L. (2009) Mechanisms of Protein Stabilization and Prevention of Protein Aggregation by Glycerol, Biochemistry. 48, 11084-11096.

38. Auton, M., Roesgen, J., Sinev, M., Holthauzen, L. M. F. \& Bolen, D. W. (2011) Osmolyte effects on protein stability and solubility: A balancing act between backbone and side-chains, Biophysical Chemistry. 159, 90-99.

39. Santoro, M. M. \& Bolen, D. W. (1992) A test of the linear extraopolation of unfolding free-energy changes over an extended denaturant concentration range, Biochemistry. 31, 4901-4907.

40. Bolen, D. W. \& Baskakov, I. V. (2001) The osmophobic effect: Natural selection of a thermodynamic force in protein folding, Journal of Molecular Biology. 310, 955-963.

41. Huecas, S., Schaffner-Barbero, C., Garcia, W., Yebenes, H., Palacios, J. M., Diaz, J. F., Menendez, M. \& Andreu, J. M. (2007) The interactions of cell division protein FtsZ with guanine nucleotides, $J$ Biol Chem. 282, 37515-28.

42. Fujita, J., Harada, R., Maeda, Y., Saito, Y., Mizohata, E., Inoue, T., Shigeta, Y. \& Matsumura, H. (2017) Identification of the key interactions in structural transition pathway of FtsZ from Staphylococcus aureus, Journal of Structural Biology. 198, 65-73.

43. Huecas, S., Marcelo, F., Perona, A., Ruiz-Avila, L. B., Morreale, A., Canada, F. J., Jimenez-Barbero, J. \& Andreu, J. M. (2015) Beyond a Fluorescent Probe: Inhibition of Cell Division Protein FtsZ by mant-GTP Elucidated by NMR and Biochemical Approaches, ACS Chem Biol. 10, 2382-2392.

44. Chemes, L. B., Alonso, L. G., Noval, M. G. \& de Prat-Gay, G. (2012) Circular dichroism techniques for the analysis of intrinsically disordered proteins and domains, Methods in molecular biology (Clifton, $\mathrm{NJ}$ ). 895, 387-404. 
FtsZ folding is induced by nucleotide binding

45. Balbach, J., Forge, V., Vannuland, N. A. J., Winder, S. L., Hore, P. J. \& Dobson, C. M. (1995) Following protein folding in real-time using NMR spectroscopy, Nature Structural Biology. 2, 865-870.

46. Wyman, J. (1964) Linked functions and reciprocalk effects in hemoglobin - a 2nd look, Advances in Protein Chemistry. 19, 223-286.

47. Kawasaki, Y. \& Freire, E. (2011) Finding a better path to drug selectivity, Drug Discovery Today. 16, 985-990.

48. Makhatadze, G. I. \& Privalov, P. L. (1996) On the entropy of protein folding, Protein Science. 5, 507510.

49. Tian, G. L., Huang, Y., Rommelaere, H., Vandekerckhove, J., Ampe, C. \& Cowan, N. J. (1996) Pathway leading to correctly folded beta-tubulin, Cell. 86, 287-296.

50. Menendez, M., Rivas, G., Diaz, J. F. \& Andreu, J. M. (1998) Control of the structural stability of the tubulin dimer by one high affinity bound magnesium ion at nucleotide $\mathrm{N}$-site, Journal of Biological Chemistry. 273, 167-176.

51. Zhang, J. \& Matthews, C. R. (1998) Ligand binding is the principal determinant of stability for the p21(H-ras) protein, Biochemistry. 37, 14881-14890.

52. Zhang, B. L., Zhang, Y. Q., Wang, Z. X. \& Zheng, Y. (2000) The role of Mg2+ cofactor in the guanine nucleotide exchange and GTP hydrolysis reactions of Rho family GTP-binding proteins, Journal of Biological Chemistry. 275, 25299-25307.

53. Burg MB \& JD, F. (2008) Intracellular organic osmolytes: function and regulation, J Biol Chem. 283, 7309-7313.

54. Whatmore, A. M., Chudek, J. A. \& Reed, R. H. (1990) Th effcets of osmotic upshock on the intracellular solute pools of Bacillus subtilis Journal of General Microbiology. 136, 2527-2535.

55. Schwan, W. R. \& Wetzel, K. J. (2016) Osmolyte transport in Staphylococcus aureus and the role in pathogenesis, World journal of clinical infectious diseases. 6, 22-27.

56. Gruendling, A. (2013) Potassium Uptake Systems in Staphylococcus aureus: New Stories about Ancient Systems, Mbio. 4.

57. Kaestle, B., Geiger, T., Gratani, F. L., Reisinger, R., Goerke, C., Borisova, M., Mayer, C. \& Wolz, C. (2015) rRNA regulation during growth and under stringent conditions in Staphylococcus aureus, Environmental Microbiology. 17, 4394-4405.

58. Sass, P., Josten, M., Famulla, K., Schiffer, G., Sahl, H. G., Hamoen, L. \& Brotz-Oesterhelt, H. (2012) Antibiotic acyldepsipeptides activate ClpP peptidase to degrade the cell division protein FtsZ, Proc Natl Acad Sci USA. 108, 17474-17479.

59. LaBreck, C. J., May, S., Viola, M. G., Conti, J. \& Camberg, J. L. (2017) The Protein Chaperone ClpX Targets Native and Non-native Aggregated Substrates for Remodeling, Disassembly, and Degradation with ClpP, Frontiers in Molecular Biosciences. 4.

60. Araujo-Bazan, L., Huecas, S., Valle, J., Andreu, D. \& Andreu, J. M. (2019) Synthetic developmental regulator MciZ targets FtsZ across Bacillus species and inhibits bacterial division, Molecular microbiology. 111, 965-980.

61. Marcelo, F., Huecas, S., Ruiz-Avila, L. B., Canada, F. J., Perona, A., Poveda, A., Martin-Santamaria, S., Morreale, A., Jimenez-Barbero, J. \& Andreu, J. M. (2013) Interactions of Bacterial Cell Division Protein FtsZ with C8-Substituted Guanine Nucleotide Inhibitors. A Combined NMR, Biochemical and Molecular Modeling Perspective, Journal of the American Chemical Society. 135, 16418-16428.

62. Rivas, G., Lopez, A., Mingorance, J., Ferrandiz, M. J., Zorrilla, S., Minton, A. P., Vicente, M. \& Andreu, J. M. (2000) Magnesium-induced linear self-association of the FtsZ bacterial cell division protein monomer - The primary steps for FtsZ assembly, Journal of Biological Chemistry. 275, 11740-11749.

63. Schuck, P. (2000) Size-distribution analysis of macromolecules by sedimentation velocity ultracentrifugation and Lamm equation modeling, Biophysical Journal. 78, 1606-1619.

64. Freyer, M. W. \& Lewis, E. A. (2008) Isothermal titration calorimetry: Experimental design, data analysis, and probing Macromolecule/Ligand binding and kinetic interactions in Biophysical Tools for Biologists: Vol 1 in Vitro Techniques (Correia, J. J. \& Detrich, H. W., eds) pp. 79-113, Elsevier Academic Press Inc, San Diego.

65. Kabsch, W. (2010) XDS, Acta Crystallographica Section D-Biological Crystallography. 66, 125-132. 
66. Winn, M. D., Ballard, C. C., Cowtan, K. D., Dodson, E. J., Emsley, P., Evans, P. R., Keegan, R. M., Krissinel, E. B., Leslie, A. G. W., McCoy, A., McNicholas, S. J., Murshudov, G. N., Pannu, N. S., Potterton, E. A., Powell, H. R., Read, R. J., Vagin, A. \& Wilson, K. S. (2011) Overview of the CCP4 suite and current developments, Acta Crystallographica Section D-Biological Crystallography. 67, 235-242.

67. McCoy, A. J., Grosse-Kunstleve, R. W., Adams, P. D., Winn, M. D., Storoni, L. C. \& Read, R. J. (2007) Phaser crystallographic software, Journal of Applied Crystallography. 40, 658-674.

68. Emsley, P., Lohkamp, B., Scott, W. G. \& Cowtan, K. (2010) Features and development of Coot, Acta Crystallographica Section D-Biological Crystallography. 66, 486-501.

69. Adams, P. D., Afonine, P. V., Bunkoczi, G., Chen, V. B., Davis, I. W., Echols, N., Headd, J. J., Hung, L.W., Kapral, G. J., Grosse-Kunstleve, R. W., McCoy, A. J., Moriarty, N. W., Oeffner, R., Read, R. J., Richardson, D. C., Richardson, J. S., Terwilliger, T. C. \& Zwart, P. H. (2010) PHENIX: a comprehensive Python-based system for macromolecular structure solution, Acta Crystallographica Section D-Biological Crystallography. 66, 213-221.

70. Lee, J. C. \& Timasheff, S. N. (1975) Reconstitution of microtubules from purified calf brain tubulin, Biochemistry. 14, 5183-5187.

Supporting Information available on-line: thermodynamic dataset Tables S1-S2, crystallographic data Table S3, NMR data Figs. S1-S2 and S5, supporting CD data Figs. S3-S4, structural images Figs. S6-S7, osmolyte screen data Fig. S8, mant-GTP titration data Fig S9 and supporting Movie 1.

Table 1: Energetics of the interactions of apo-SaFts $\mathrm{Z}_{\mathrm{T}}$ with guanine nucleotides in $25 \mathrm{mM}$ Pipes$\mathrm{KOH}, 1 \mathrm{mM}$ EDTA, $\mathrm{pH}$ 7.5, with glycerol and $\mathrm{KCl}$, determined by $\mathrm{ITC}$ at $25^{\circ} \mathrm{C}^{\mathrm{a}}$.

\begin{tabular}{|c|c|c|c|c|c|c|}
\hline & Ligand & $K_{D}(\mathbf{n M})$ & $\begin{array}{c}\Delta G \\
(\mathrm{kcal} / \mathrm{mol})\end{array}$ & $\begin{array}{c}\Delta H \\
(\mathrm{kcal} / \mathrm{mol})\end{array}$ & $\begin{array}{c}-\mathrm{T} \Delta \mathrm{S} \\
(\mathrm{kcal} / \mathrm{mol})\end{array}$ & $\mathbf{n}$ \\
\hline \multirow{4}{*}{$\begin{array}{l}3.4 \mathrm{M} \text { glycerol, } \\
0.05 \mathrm{M} \mathrm{KCl}\end{array}$} & GTP & $19.5 \pm 5.5$ & $-10.5 \pm 0.1$ & $-11.8 \pm 0.9$ & $1.2 \pm 1.0$ & $0.84 \pm 0.04$ \\
\hline & GDP & $18.2 \pm 2.8$ & $-10.6 \pm 0.2$ & $-13.3 \pm 0.1$ & $2.7 \pm 0.2$ & $0.64 \pm 0.01$ \\
\hline & GDP- $\mathrm{Mg}^{2+}$ & $251 \pm 25$ & $-9.0 \pm 0.2$ & $-16.9 \pm 0.2$ & $7.9 \pm 0.3$ & $0.57 \pm 0.01$ \\
\hline & mant-GTP & $217 \pm 40$ & $-9.1 \pm 0.1$ & $-9.7 \pm 0.3$ & $0.6 \pm 0.3$ & $0.65 \pm 0.04$ \\
\hline \multirow[t]{2}{*}{$0.35 \mathrm{M} \mathrm{KCl}$} & GTP & $194 \pm 15$ & $-9.1 \pm 0.1$ & $-9.4 \pm 0.1$ & $0.3 \pm 0.1$ & $0.71 \pm 0.01$ \\
\hline & mant-GTP & $25700 \pm 5600$ & $-6.3 \pm 0.1$ & $-4.7 \pm 0.5$ & $-1.5 \pm 0.5$ & $0.86 \pm 0.05$ \\
\hline 3.4 M glycerol, & GTP & $183 \pm 50$ & $-9.2 \pm 0.2$ & $-9.0 \pm 0.4$ & $-0.2 \pm 0.5$ & $0.79 \pm 0.03$ \\
\hline & mant-GTP & $47000 \pm 18000$ & $-5.9 \pm 0.5$ & $-15.3 \pm 15.4$ & $9.4 \pm 15.4$ & $0.49 \pm 0.40$ \\
\hline
\end{tabular}

${ }^{\mathrm{a}}$ Values are average and standard error of two determinations. No binding could be measured in $0.05 \mathrm{M} \mathrm{KCl}$ in the absence of glycerol.

\section{FIGURE LEGEND}

Figure 1. Structural analysis SaFtsZ in solution with and without bound nucleotide. A. CD spectra of $20 \mu \mathrm{M} \mathrm{SaFtsZ}$ f plus $1 \mathrm{mM} \mathrm{GTP}$ (red line), SaFtsZ $\mathrm{wt}_{\mathrm{wt}}$ plus $1 \mathrm{mM} \mathrm{GTP}$ (gray), SaFtsZ plus $1 \mathrm{mM}$ GTP (green), apo-SaFtsZ $\mathrm{T}_{\mathrm{T}}$ without nucleotide (black) and SaFtsZ $\mathrm{wt}_{\mathrm{wt}}$ in $6 \mathrm{M} \mathrm{GdnCl}$ (dark yellow). Inset: sedimentation coefficient distribution $\mathrm{c}(\mathrm{S})$ of $19 \mu \mathrm{M}$ apo-SaFtsZ (black $_{\mathrm{T}}$ line; $\mathrm{s}_{20, \mathrm{w}}=2.00 \mathrm{~S}, \mathrm{f} / \mathrm{fmin}=1.82$ from sedimentation-diffusion analysis, apparent $\mathrm{Mw} \approx 32200$ Da, calc. Mw $31900 \mathrm{Da}$ ), $19 \mu \mathrm{M} \mathrm{SaFtsZ} \mathrm{T}_{\mathrm{T}}$ (green; $\mathrm{s}_{20, \mathrm{w}}=2.78 \mathrm{~S}$ f/fmin $=1.27$, app. $\mathrm{Mw} \approx 30900$ 
FtsZ folding is induced by nucleotide binding Da, calc. Mw 32400 Da including GTP) and $15 \mu \mathrm{M} \mathrm{SaFtsZ}_{\mathrm{f}}\left(\right.$ red; $\mathrm{s}_{20, \mathrm{w}}=2.77 \mathrm{~S}, \mathrm{f} / \mathrm{fmin}=1.66$, app. $\mathrm{Mw} \approx 45600 \mathrm{Da}$, calc. $41900 \mathrm{Da}$ including GTP). B. Downfield region of $1 \mathrm{D}{ }^{1} \mathrm{H}$ NMR spectra of SaFtsZ in deuterated buffer: $431 \mu \mathrm{M} \mathrm{SaFtsZ}$ f plus $1 \mathrm{mM} \mathrm{GTP}$ (red), $600 \mu \mathrm{M} \mathrm{SaFtsZ}$ plus $1 \mathrm{mM}$ GTP (green), $260 \mu \mathrm{M}$ apo-SaFtsZ $\mathrm{T}_{\mathrm{T}}$ without nucleotide (black). C. Upfield region of the same spectra of apo-SaFtsZ $\mathrm{T}_{\mathrm{T}}, \mathrm{SaFts}_{\mathrm{T}}$ and $\mathrm{SaFts}_{\mathrm{f}}$. The inset (upper right) shows a magnified view of the upfield spectral region highlighting the appearance of peaks whose $\delta<0.6 \mathrm{ppm}$, which are hallmarks of folded protein. The complete spectra are shown in Fig. S1. For sets of spectra of SaFtsZ $\mathrm{T}$ and SaFtsZ $\mathrm{f}$ at different temperatures, see Fig. S2.

Figure 2. Nucleotide-induced assembly of SaFtsZ. A. Polymerization, monitored by light scattering, was triggered by addition of $50 \mu \mathrm{M}$ GMPCPP. The traces correspond to $15 \mu \mathrm{M}$

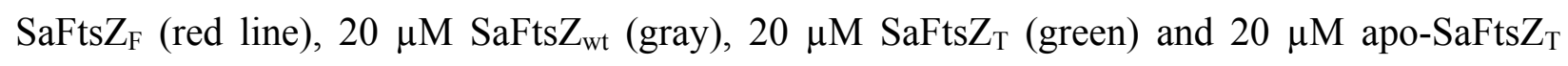
(black); $20 \mu \mathrm{M}$ apo-SaFtsZ T $_{\mathrm{T}}$ with $1 \mathrm{mM}$ GTP gave less scattering (dark yellow line, 5x amplified). B. Polymer sedimentation electrophoretic assay of SaFtsZ (1 g/L) with different guanine nucleotides (1 mM each). S, supernatant; P, pellet. The numbers below indicate percent of protein in pellet. C. Representative electron micrographs (negative stain) of the types of FtsZ polymers formed.

Figure 3. Apo-SaFtsZ $Z_{T}$ folding induced by glycerol and GTP. A. CD spectra of apo-SaFtsZ (gray dashed line) with increasing glycerol percentages (v/v): $5 \%$ (black) $10 \%$ (blue), $15 \%$ (light blue), $20 \%$ (pink), $30 \%$ (red), $40 \%$ (dark red), and in $6 \mathrm{M} \mathrm{GdnCl} \mathrm{(dark} \mathrm{yellow).} \mathrm{Inset,}$ change in ellipticity at $222 \mathrm{~nm}$ with glycerol concentration. The line is least-squares fit of a twostate model equilibrium between unfolded (U) and folded (F) states (Methods). $\Delta \mathrm{G}_{\text {fold, }}$ oM glycerol $=$ $2.62 \pm 0.21 \mathrm{kcal} \mathrm{mol}^{-1}$, osmolyte efficiency $m=1.31 \pm 0.10 \mathrm{kcal} \mathrm{mol}^{-1} \mathrm{M}^{-1}$, ellipticity (min) = $3250 \pm 110$, ellipticity $(\max )=-9380 \pm 70, \mathrm{r}^{2}=0.999$. B. CD spectra of apo-SaFtsZ $\mathrm{T}_{\mathrm{T}}$ with increasing GTP concentrations: $20 \mu \mathrm{M}$ (dark blue line), $100 \mu \mathrm{M}$ (blue), $250 \mu \mathrm{M}$ (light blue), 500 $\mu \mathrm{M}$ (green), $1000 \mu \mathrm{M}$ (red). Inset, change in ellipticity at $222 \mathrm{~nm}$ with GTP concentration measured in several experiments; the points scatter is inherent to noise from GTP light absorption and the slow rate of the CD change. The line is a least squares fit of a GTP (G) facilitated folding mechanism indicated, neglecting GTP binding to the unfolded protein. For this reaction scheme, $K_{\text {fold, obsd }}=\mathrm{K}_{1}\left(1+\mathrm{K}_{2}[\mathrm{G}]\right)$. Substituting the $\mathrm{K}_{1}$ value from the folding analysis above $\left(\mathrm{K}_{1}=\mathrm{K}_{\text {fold, } 0 \mathrm{M} \text { glycerol }}=0.0120\right)$ permitted the determination of best fit values of the dissociation constant $\mathrm{K}_{\mathrm{D} 2}=\mathrm{K}_{2}{ }^{-1}=126 \pm 41 \mathrm{nM}$, ellipticity (min) $=-3400 \pm 340$, ellipticity $(\max )=-9580 \pm 390, \mathrm{r}^{2}=0.94$. All CD spectra were acquired after 120 min incubation of 20 $\mu \mathrm{M}$ protein in buffer at $25{ }^{\circ} \mathrm{C}$. C. Kinetics of $\mathrm{FtsZ}_{\mathrm{T}}$ refolding followed by $1 \mathrm{D}{ }^{1} \mathrm{H}$ NMR 
spectroscopy. Left axis, squares, integral of denaturated peak region $(1.02-0.80 \mathrm{ppm}$, red squares); right axis, integrals of native peaks: $0.68-0.52 \mathrm{ppm}=$ blue triangles, $0.51-0.38=$ green circles, ${ }^{-} 0.03-{ }^{-} 0.17 \mathrm{ppm}=$ pink diamonds. Dotted lines show the fits of a single exponential equation to the data. The values obtained from the fits are: $\mathrm{k}_{\mathrm{obs}}=0.019 \pm 0.003 \mathrm{~min}^{-}$ ${ }^{1}, \mathrm{R}=0.958$ (squares), $\mathrm{k}_{\text {obs }}=0.017 \pm 0.003 \mathrm{~min}^{-1}, \mathrm{R}=0.948$ (triangles); $\mathrm{k}_{\text {obs }}=0.015 \pm 0.004$ $\min ^{-1}, \mathrm{R}=0.897$ (circles); $\mathrm{k}_{\mathrm{obs}}=0.015 \pm 0.003 \mathrm{~min}^{-1}, \mathrm{R}=0.935$ (diamonds). The source NMR spectra are shown in Fig. S5.

Figure 4. Nucleotide binding by apo-SaFts $Z_{T}$. A. ITC titration of GTP binding to apo-SaFtsZ in $25 \%(\mathrm{v} / \mathrm{v})$ glycerol. B. GTP binding in 5\% glycerol. C. Mant-GTP binding in 25\% glycerol. Thermograms (top) and binding isotherms (bottom) are shown in each case. Solid lines are the best model fits to experimental parameters. D. Dependence of the apparent GTP binding affinity $\left(\mathrm{K}_{\mathrm{b} \text {, obsd }}\right)$ on glycerol concentration (filled circles). Notice that according to the ligand facilitated folding model indicated, $\mathrm{K}_{\mathrm{b}, \mathrm{bbsd}}=\mathrm{K}_{1} \mathrm{~K}_{2} /\left(1+\mathrm{K}_{1}\right)$. Substituting the $\mathrm{K}_{1}$ value calculated at each glycerol concentration from the folding analysis in Figure 3A (inset), permitted us to calculate the values of binding affinity to the folded protein $\mathrm{K}_{2}$ (void circles). The lines are sigmoid curve fits drawn only to show the trend of the data. E. Thermodynamic signature plots for GTP binding at high and low glycerol concentrations, showing $\Delta \mathrm{G}$ (blue bars), $\Delta \mathrm{H}$ (green) and $-\mathrm{T} \Delta \mathrm{S}$ (red).

Figure 5. X-ray crystallography characterization of SaFtsZ $\mathbf{T}_{\mathrm{T}_{\dot{.}}}$ A. Schematic representation of SaFtsZ, with boxes representing $\alpha$-helices $(\mathrm{H})$ and $\beta$-strands (S) and nucleotide-binding loops (T) indicated below; dashed lines indicate disordered segments. B. Canonical front view of the refolded apo-SaFtsZ $\mathrm{T}_{\mathrm{T}}$ in complex with GDP, using the same nomenclature and color scheme as in panel A. C. A cavity behind the NBS is occupied by six water molecules in SaFtsZ $\mathrm{T}^{-}$-Wat or ethylene glycol in SaFtsZ $\mathrm{T}_{\mathrm{T}}$-EthGly (Fig. S6). D. Canonical front view of the SaFts $\mathrm{T}_{\mathrm{T}}-\mathrm{Apo}_{\text {folded }}$ state, using the same nomenclature and color scheme as in panels A and B (Fig. S7).

Figure 6. Fluorescence assays of ligand binding to the nucleotide site of FtsZ. A. Displacement of mant-GTP $(500 \mathrm{nM})$ from apo-SaFts $\mathrm{T}_{\mathrm{T}}\left(250 \mathrm{nM}\right.$ binding sites; $\mathrm{K}_{\mathrm{D}}=0.57 \pm 0.08$ $\mu \mathrm{M}$ ), measured by fluorescence anisotropy, by GTP (gray circles, $K_{\mathrm{D}}$ app $3.3 \mathrm{nM}$ ) and the synthetic compounds UCM44 (black circles, $\mathrm{K}_{\mathrm{D} \text { app }} 26 \mu \mathrm{M}$ ) and UCM81 (triangles, $\mathrm{K}_{\mathrm{D} \text { app }} 5.9$ $\mu \mathrm{M})$. Solid lines are best fits to the data according to a single site competition model. B. Similar competition experiments performed with apo-EcFtsZ (400 nM binding sites) and mant-GTP (500 $\mathrm{nM}$; a $\mathrm{K}_{\mathrm{D}}$ value $0.8 \mu \mathrm{M}$ was employed), showing displacement data by GDP (gray circles, $\mathrm{K}_{\mathrm{D} \text { app }}$ $45 \mathrm{nM})$, UCM44 (K $\left.\mathrm{K}_{\mathrm{D} \text { app. } ~} 140 \mu \mathrm{M}\right)$ and UCM81. 
Figure 1

\section{A}
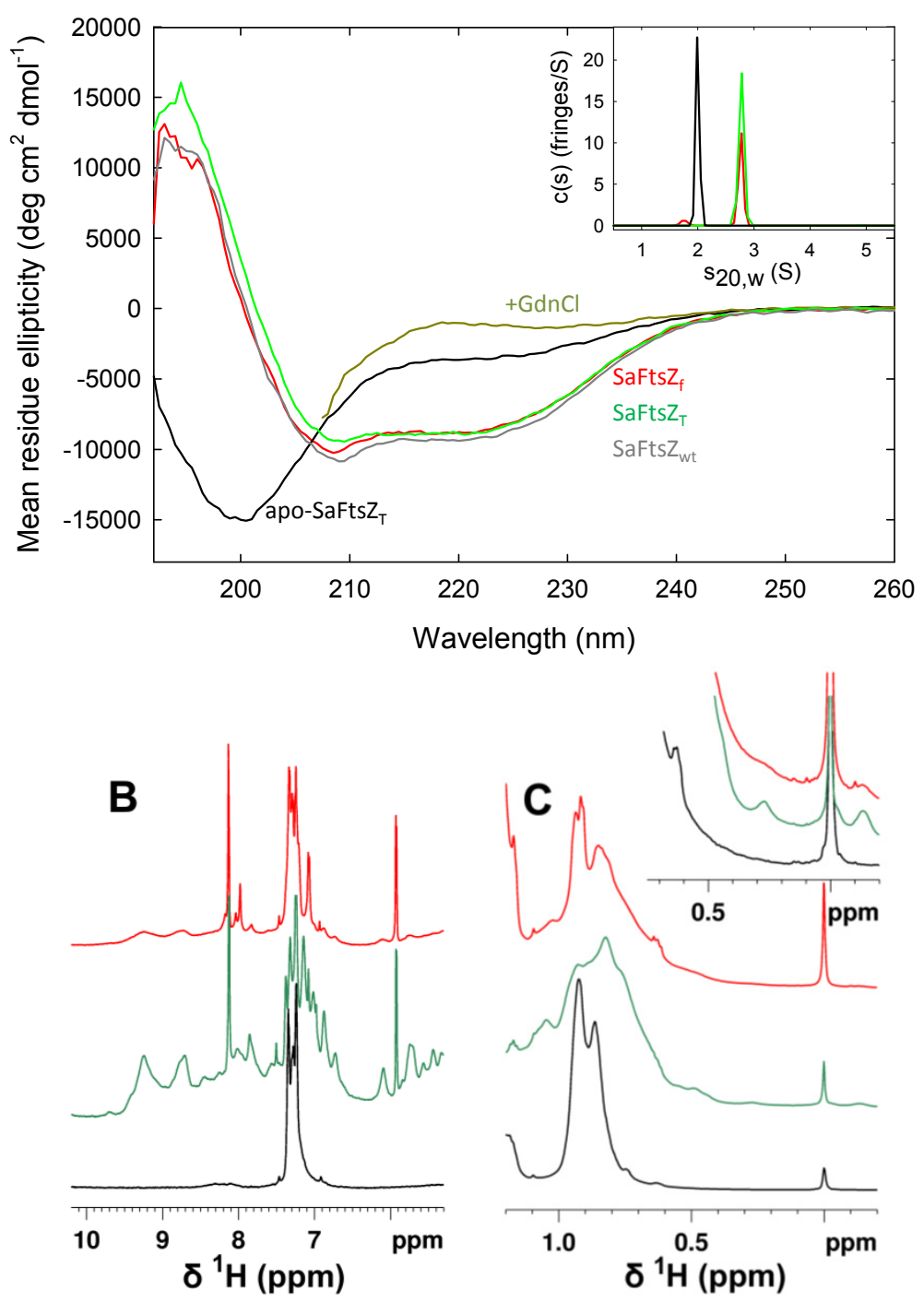
Figure 2

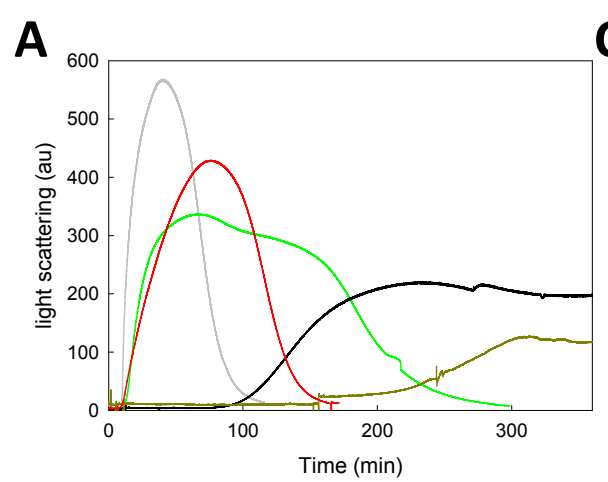

B
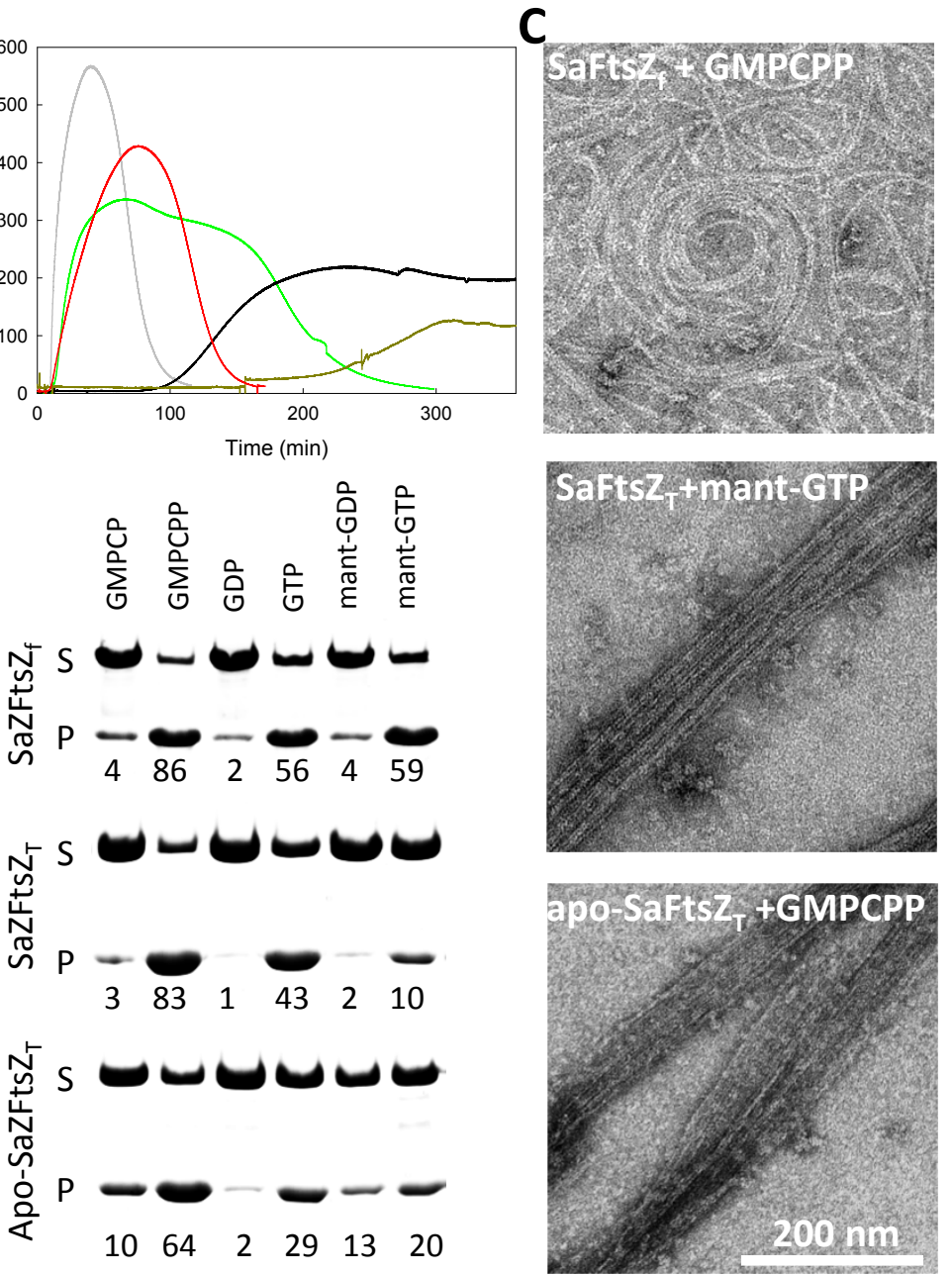
Figure 3

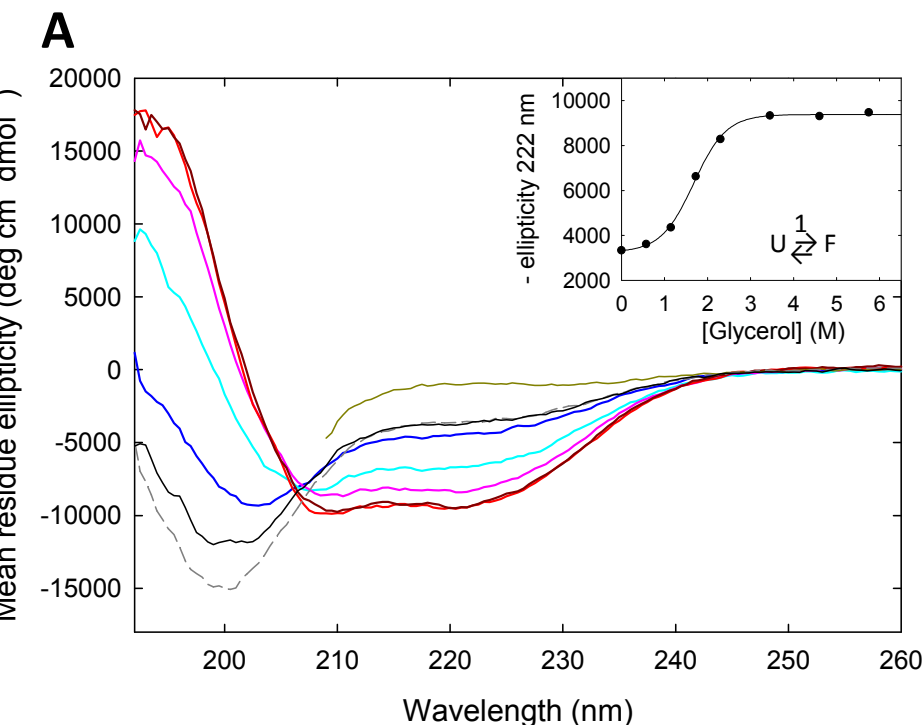

B

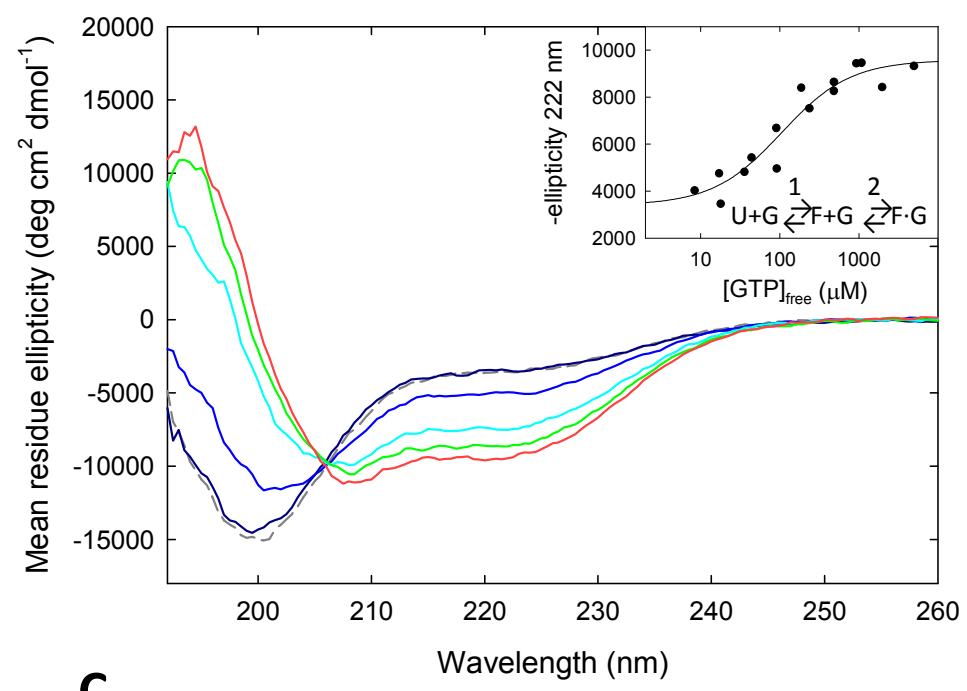

C

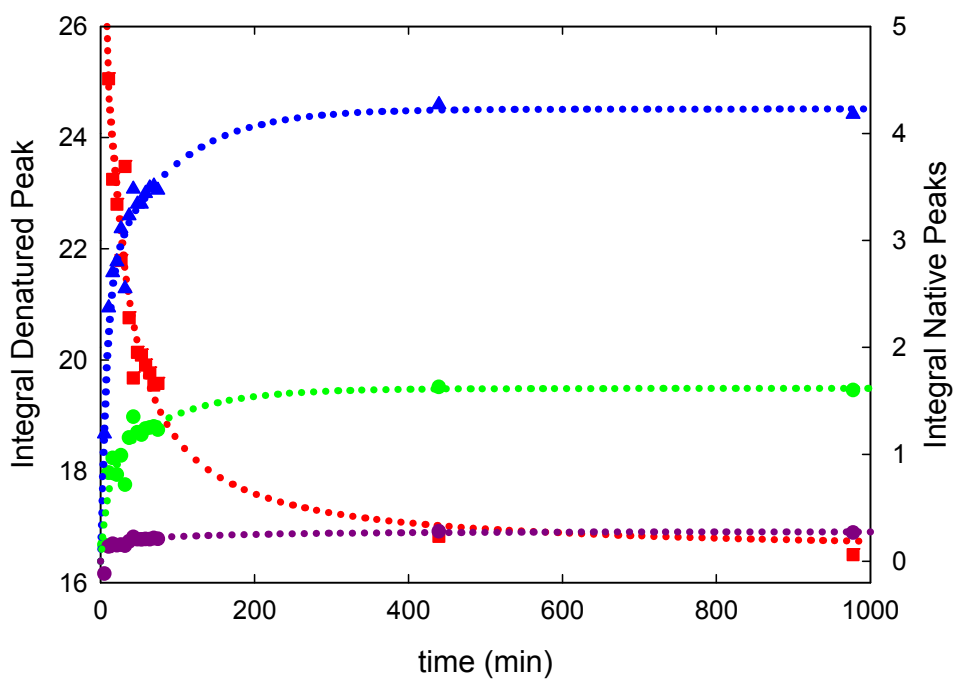


Figure 4
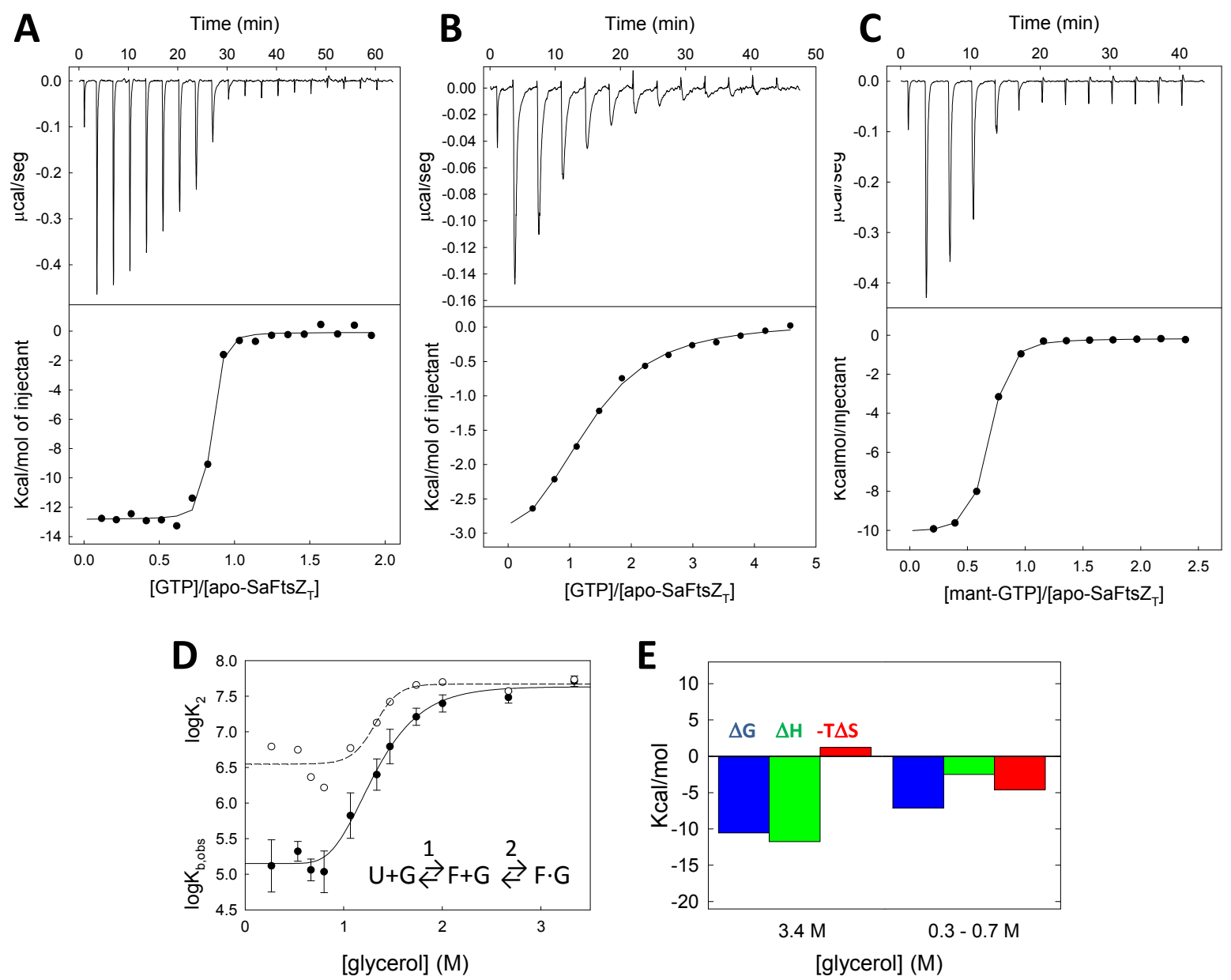
Figure 5
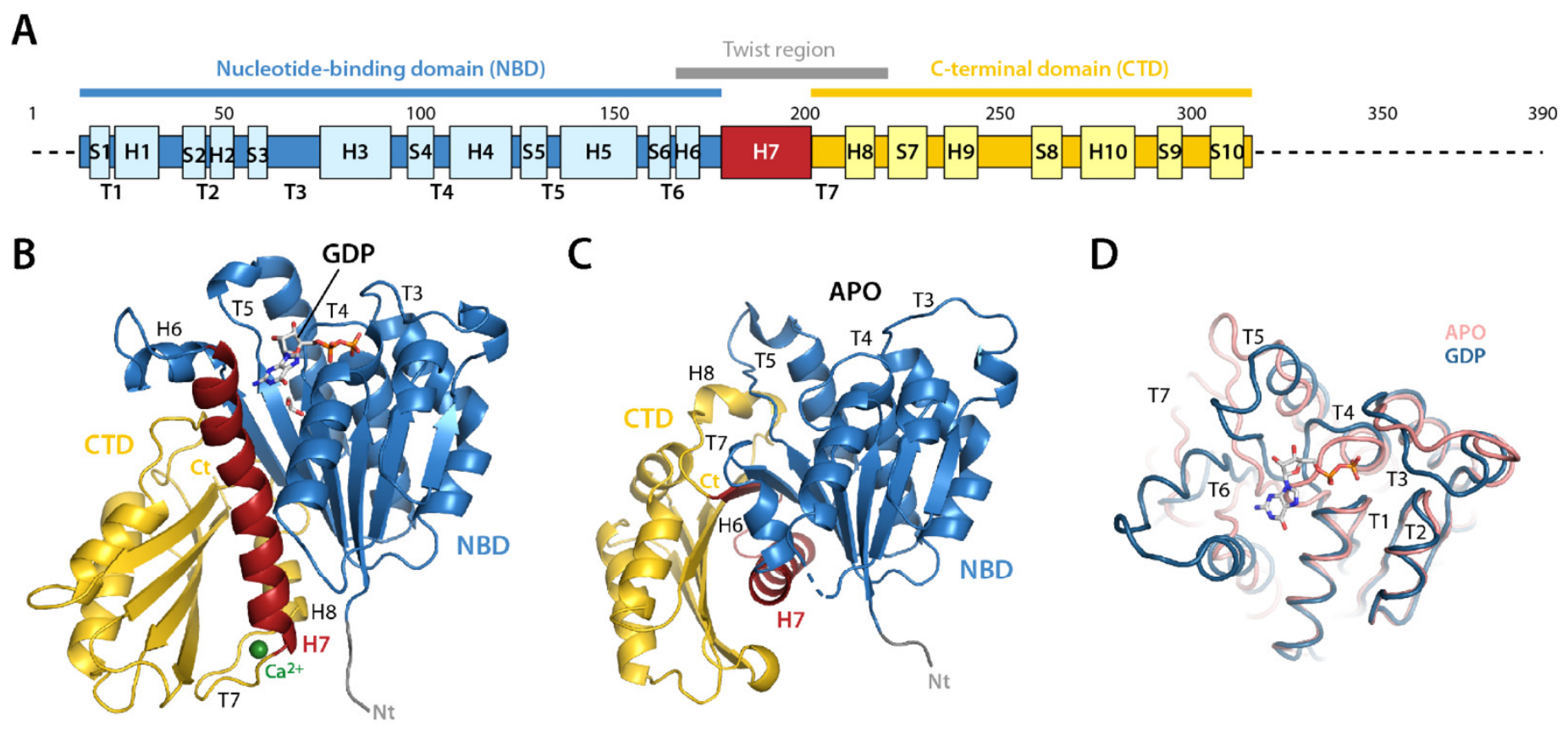

B

E

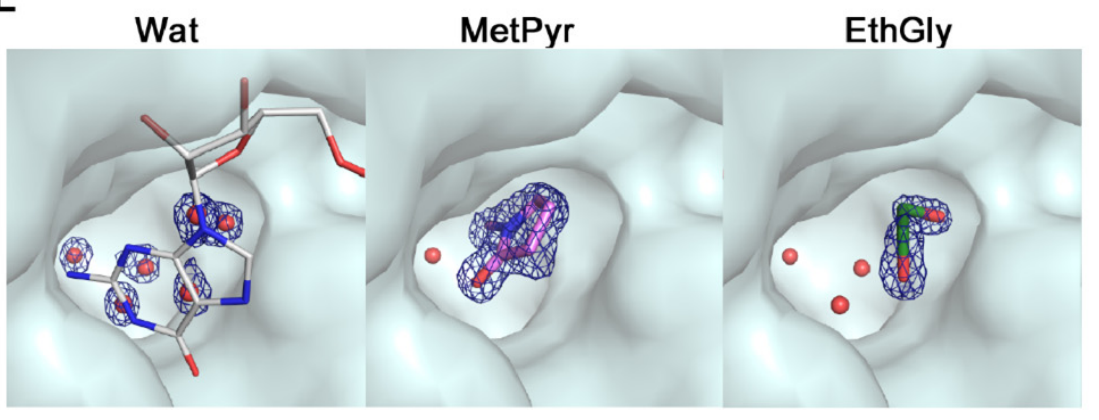

C

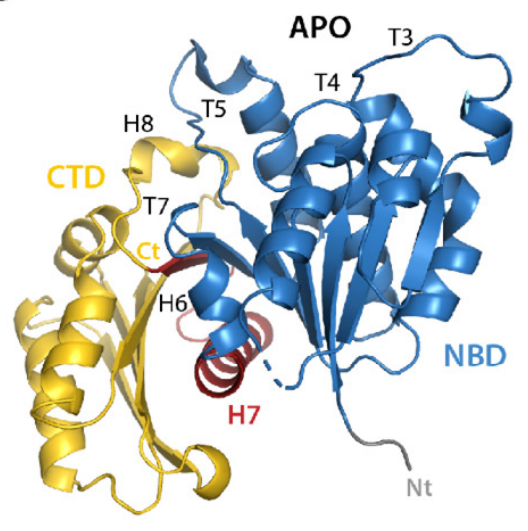

F
D

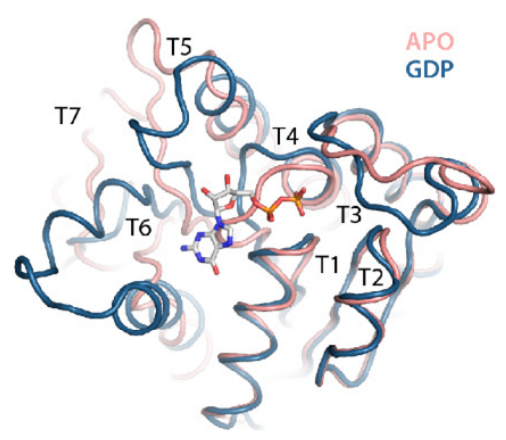

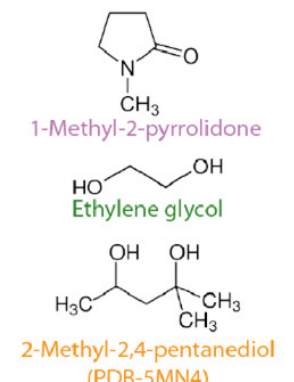

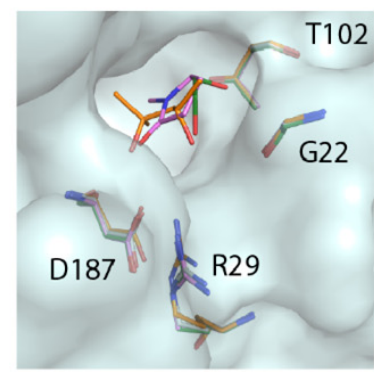


Figure 6
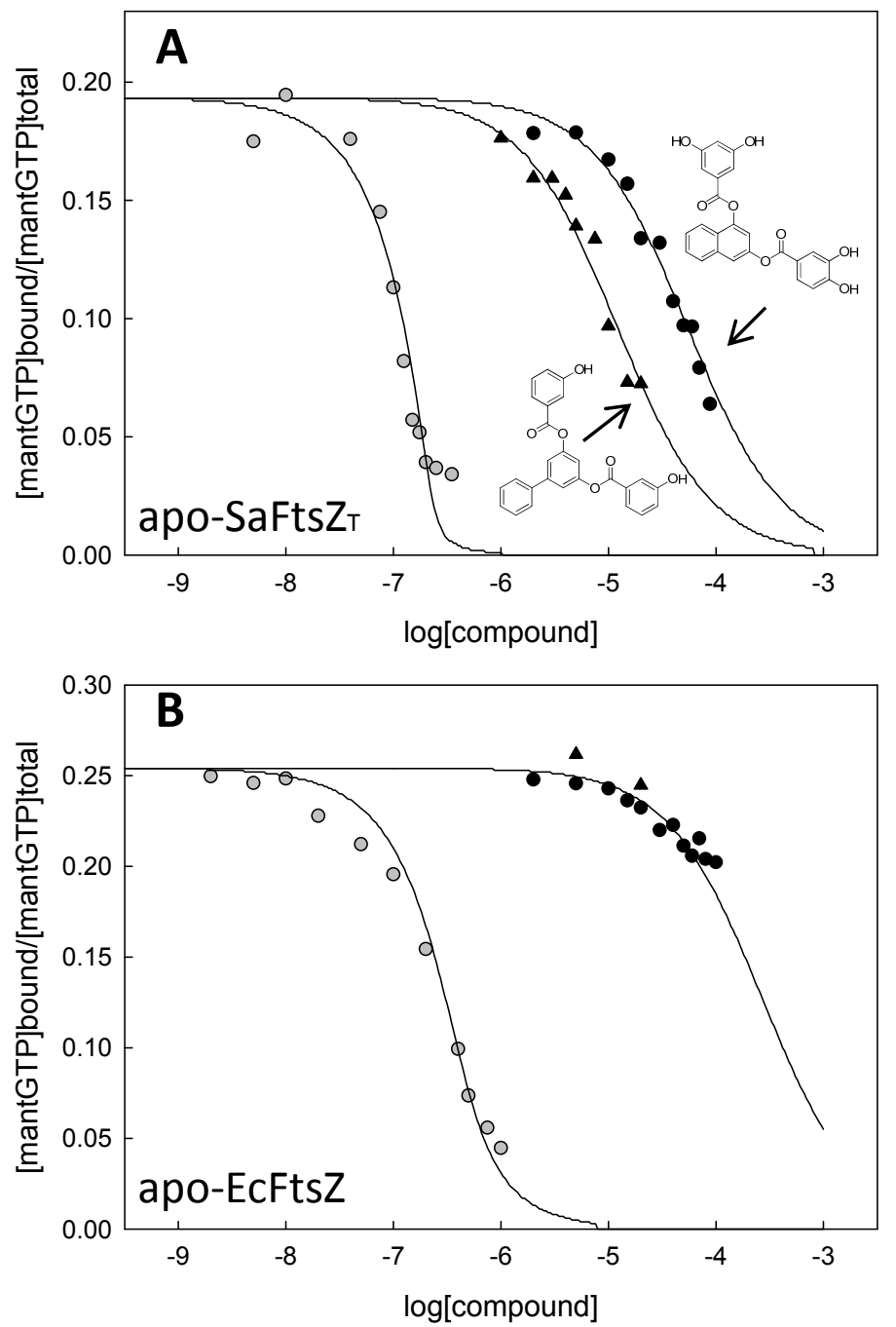


\section{Supporting Information}

\section{Nucleotide-induced folding of cell division protein FtsZ from Staphylococcus aureus}

Sonia Huecas, Alejandro J. Canosa-Valls, Lidia Araújo-Bazán, Federico M. Ruiz,

Douglas V. Laurents, Carlos Fernández-Tornero, and José M. Andreu

\section{TABLES}

Table S1. Thermodynamic data of the GTP apo-SaFts $Z_{\mathrm{T}}$ interaction at different $\mathrm{KCl}$ concentrations, in $25 \mathrm{mM}$ Pipes-KOH, $1 \mathrm{mM}$ EDTA, pH 7.5 measured by ITC at $25^{\circ} \mathrm{C}^{\mathrm{a}}$.

\begin{tabular}{|c|c|c|c|c|c|}
\hline$[\mathrm{KCl}](\mathbf{M})$ & $\mathbf{K}_{\mathbf{D}}(\mathbf{n M})$ & $\boldsymbol{\Delta G}(\mathbf{k c a l} / \mathbf{m o l})$ & $\mathbf{\Delta H}(\mathbf{k c a l} / \mathbf{m o l})$ & $\mathbf{- T \Delta S}(\mathbf{k c a l} / \mathbf{m o l})$ & $\mathbf{n}$ \\
\hline 0.20 & $220 \pm 50$ & $-9.1 \pm 0.1$ & $-8.3 \pm 0.2$ & $-0.8 \pm 0.2$ & $0.81 \pm 0.02$ \\
\hline 0.35 & $194 \pm 15$ & $-9.1 \pm 0.1$ & $-9.4 \pm 0.1$ & $0.3 \pm 0.1$ & $0.71 \pm 0.01$ \\
\hline 0.60 & $645 \pm 70$ & $-8.4 \pm 0.1$ & $-8.5 \pm 0.2$ & $0.1 \pm 0.2$ & $0.79 \pm 0.01$ \\
\hline 1.00 & $1600 \pm 100$ & $-7.9 \pm 0.1$ & $-7.4 \pm 0.1$ & $-0.5 \pm 0.1$ & $0.67 \pm 0.01$ \\
\hline
\end{tabular}

${ }^{a}$ Values are average and standard error of two experiments

Table S2. Thermodynamic data of the GTP-FtsZ interactions at different glycerol concentrations, in $25 \mathrm{mM}$ Pipes-KOH, $50 \mathrm{mM} \mathrm{KCl}, 1 \mathrm{mM}$ EDTA, pH 7.5, measured by ITC at $25^{\circ} \mathrm{C}^{\mathrm{a}}$.

\begin{tabular}{|c|c|c|c|c|c|c|}
\hline & $\begin{array}{c}\text { [Glycerol] } \\
\text { (M) }\end{array}$ & $\mathrm{K}_{\mathrm{D}}(\mathrm{nM})$ & $\Delta G($ kcal $/ \mathrm{mol})$ & $\Delta \mathrm{H}(\mathrm{kcal} / \mathrm{mol})$ & $-\mathrm{T} \Delta \mathrm{S}$ (kcal/mol) & $\mathbf{n}$ \\
\hline \multirow{11}{*}{$\begin{array}{l}\text { Apo- } \\
\text { SaFtsZ }\end{array}$} & 3.4 & $19.5 \pm 5.5$ & $-10.5 \pm 0.1$ & $-11.8 \pm 0.9$ & $1.2 \pm 1.0$ & $0.84 \pm 0.04$ \\
\hline & 2.7 & $32.9 \pm 6.9$ & $-10.2 \pm 0.2$ & $-17.2 \pm 0.2$ & $7.0 \pm 0.4$ & $0.69 \pm 0.05$ \\
\hline & 2.0 & $40 \pm 10$ & $-10.1 \pm 0.5$ & $-11.4 \pm 0.2$ & $1.3 \pm 0.5$ & $0.82 \pm 0.10$ \\
\hline & 1.7 & $62 \pm 16$ & $-9.8 \pm 0.6$ & $-19.2 \pm 0.4$ & $9.3 \pm 0.7$ & $0.55 \pm 0.10$ \\
\hline & 1.5 & $161 \pm 55$ & $-9.3 \pm 0.2$ & $-13.4 \pm 0.5$ & $4.1 \pm 0.7$ & $0.84 \pm 0.20$ \\
\hline & 1.3 & $399 \pm 114$ & $-8.7 \pm 0.2$ & $-12.0 \pm 0.4$ & $3.3 \pm 0.5$ & $0.72 \pm 0.10$ \\
\hline & 1.0 & $1500 \pm 700$ & $-8.0 \pm 0.3$ & $-15.4 \pm 2.1$ & $7.4 \pm 2.4$ & $0.65 \pm 0.10$ \\
\hline & 0.8 & $9200 \pm 4000$ & $-6.9 \pm 0.2$ & $-11.0 \pm 0.3$ & $4.0 \pm 0.5$ & $0.63 \pm 0.04$ \\
\hline & 0.7 & $8670 \pm 1720$ & $-6.9 \pm 0.3$ & $-3.8 \pm 0.3$ & $-3.1 \pm 0.4$ & $1.24 \pm 0.20$ \\
\hline & 0.5 & $4760 \pm 770$ & $-7.3 \pm 0.4$ & $-4.4 \pm 0.3$ & $-2.9 \pm 0.5$ & $0.83 \pm 0.10$ \\
\hline & 0.3 & $7600 \pm 5000$ & $-7.1 \pm 0.4$ & $-0.9 \pm 0.2$ & $-6.2 \pm 0.6$ & $1.74 \pm 0.10$ \\
\hline \multirow[t]{2}{*}{ BsFtsZ } & 3.4 & $12.4 \pm 1.0$ & $-10.8 \pm 0.1$ & $-4.2 \pm 0.4$ & $-6.6 \pm 0.4$ & $1.01 \pm 0.20$ \\
\hline & 0 & $349 \pm 76$ & $-8.8 \pm 0.1$ & $-6.3 \pm 1.3$ & $-2.5 \pm 1.4$ & $0.77 \pm 0.30$ \\
\hline \multirow{2}{*}{$\begin{array}{l}\text { Apo- } \\
\text { MjFtsZ }\end{array}$} & 3.4 & $4.4 \pm 1.5$ & $-11.5 \pm 0.2$ & $-4.6 \pm 0.2$ & $-6.9 \pm 0.3$ & $0.81 \pm 0.04$ \\
\hline & 0 & $10.3 \pm 3.0$ & $-10.9 \pm 0.1$ & $-6.7 \pm 0.1$ & $-4.2 \pm 0.1$ & $0.91 \pm 0.02$ \\
\hline
\end{tabular}

${ }^{a}$ Values are average and standard error of two to four experiments. $\Delta \Delta \mathrm{G}_{\mathrm{b} \text { Bs }}(0->3.4 \mathrm{M}$ glycerol $)=-2.0 \pm 0.1 \mathrm{kcal} / \mathrm{mol}$, $\Delta \Delta \mathrm{G}_{\mathrm{b} \text { Mj }}(0->3.4 \mathrm{M}$ glycerol $)=-0.6 \pm 0.2 \mathrm{kcal} / \mathrm{mol}$. 
Table S3. Crystallographic data collection and refinement statistics.

\begin{tabular}{|c|c|c|c|c|c|}
\hline & SaFtsZ-refold & SaFtsZ-Apo & SaFtsZ-GDP-Wat & SaFtsZ-GDP-MetPyr & SaFtsZ-GDP-EthGly \\
\hline Crystallization & $\begin{array}{r}25 \% \text { PEG5K MME, } 10 \% \\
\text { EthGly, } 0.2 \mathrm{M} \mathrm{Li}_{2} \mathrm{SO}_{4}, \\
0.1 \mathrm{M} \text { Tris } \mathrm{pH} 9.0\end{array}$ & $\begin{array}{r}2.1 \mathrm{M}\left(\mathrm{NH}_{4}\right)_{2} \mathrm{SO}_{4}, \\
0.1 \mathrm{M} \mathrm{MES} \mathrm{pH} 6.2 ; \\
\text { cryo } 30 \% \text { glycerol }\end{array}$ & $\begin{array}{l}25 \% \text { PEG5K MME, } 10 \% \\
\text { EthGly, } 0.2 \mathrm{M} \mathrm{Li} \mathrm{LO}_{2}, \\
0.1 \mathrm{M} \mathrm{Tris} \mathrm{pH} 8.5\end{array}$ & $\begin{array}{l}26 \% \text { PEG5K MME, } 10 \% \\
\text { EthGly, } 0.2 \mathrm{M} \mathrm{Li}_{2} \mathrm{SO}_{4} \\
0.1 \mathrm{M} \text { Tris pH } 8.7\end{array}$ & $\begin{array}{l}27.5 \% \text { PEG5K MME, } 10 \% \\
\text { EthGly, } 0.2 \mathrm{M} \mathrm{Li}_{2} \mathrm{SO}_{4} \\
0.1 \mathrm{M} \text { Tris } \mathrm{pH} 9.0\end{array}$ \\
\hline \multicolumn{6}{|l|}{ Data collection } \\
\hline Wavelength $(\AA)$ & 0.9793 & 0.9795 & 0.9793 & 0.9790 & 0.9793 \\
\hline Space group & $\mathrm{C} 2$ & $\mathrm{P} 2{ }_{1} 2_{1} 2_{1}$ & $\mathrm{C} 2$ & $\mathrm{C} 2$ & C2 \\
\hline Unit cell $\left(\AA,{ }^{0}\right)$ & $\begin{array}{r}72.7,50.1,88.7 \\
90,111.7,90 \\
41.20-1.90(1.97-1.90)\end{array}$ & $\begin{array}{r}72.6,78.8,225.6 \\
90,90,90 \\
40.00-2.15(2.27-2.15)\end{array}$ & $\begin{array}{r}71.7,51.3,88.2 \\
90,110.9,90 \\
40.00-1.24(1.31-1.24)\end{array}$ & $\begin{array}{r}72.5,50.1,88.5 \\
90,111.8,90 \\
40.0-1.16(1.22-1.16)\end{array}$ & $\begin{array}{r}72.4,50.5,88.4 \\
90,111.5,90 \\
40.0-1.14(1.20-1.14)\end{array}$ \\
\hline Unique reflections & $22836(2265)$ & $70836(10226)$ & $84186(12233)$ & $92535(8143)$ & $108093(15595)$ \\
\hline Multiplicity & $1.9(1.9)$ & $5.5(5.7)$ & $6.4(6.4)$ & $3.7(2.8)$ & $3.3(3.2)$ \\
\hline Completeness (\%) & $96.8(96.5)$ & $99.9(100)$ & $99.9(100)$ & $91.0(55.1)$ & $99.1(98.1)$ \\
\hline Mean I/sigma(I) & $7.5(1.4)$ & $13.6(1.5)$ & $8.2(1.6)$ & $13.4(0.8)$ & $11.5(1.2)$ \\
\hline R-merge & $0.055(0.417)$ & $0.057(1.022)$ & $0.119(1.414)$ & $0.033(0.968)$ & $0.040(0.828)$ \\
\hline R-pim & $0.055(0.417)$ & $0.026(0.469)$ & $0.050(0.608)$ & $0.019(0.682)$ & $0.026(0.543)$ \\
\hline $\mathrm{CC} 1 / 2$ & $0.995(0.804)$ & $0.998(0.662)$ & $0.993(0.710)$ & $0.999(0.635)$ & $0.998(0.716)$ \\
\hline Wilson B-factor & 28.93 & 58.89 & 17.50 & 17.50 & 16.82 \\
\hline \multicolumn{6}{|l|}{ Refinement } \\
\hline Reflections (Total / R-free) & $22815 / 1090$ & $70613 / 3523$ & $83805 / 3981$ & $92478 / 4732$ & $107938 / 5411$ \\
\hline$R / R$-free & $0.173 / 0.238$ & $0.229 / 0.255$ & $0.195 / 0.210$ & $0.191 / 0.208$ & $0.207 / 0.212$ \\
\hline Non-hydrogen atoms & 2469 & 8717 & 2440 & 2489 & 2550 \\
\hline Macromolecule & 2223 & 8575 & 2221 & 2221 & 2221 \\
\hline Ligand & 33 & 67 & 29 & 36 & 33 \\
\hline Solvent & 213 & 75 & 190 & 232 & 296 \\
\hline Protein residues & 307 & 1192 & 310 & 310 & 310 \\
\hline Bond length rmsd ( $(\AA)$ & 0.0006 & 0.002 & 0.019 & 0.009 & 0.008 \\
\hline Bond angle rmsd $\left({ }^{0}\right)$ & 0.88 & 0.45 & 1.68 & 1.11 & 1.11 \\
\hline Ramachandran favoured (\%) & 98.36 & 98.89 & 99.02 & 99.67 & 99.67 \\
\hline Ramachandran allowed (\%) & 1.64 & 1.11 & 0.98 & 0.33 & 0.33 \\
\hline Ramachandran outliers (\%) & 0.00 & 0.00 & 0.00 & 0.00 & 0.00 \\
\hline Rotamer outliers (\%) & 0.00 & 0.33 & 0.43 & 0.86 & 0.86 \\
\hline Clashscore & 4.65 & 4.26 & 3.33 & 3.32 & 4.21 \\
\hline Average B-factor $\left(\AA^{2}\right)$ & 36.32 & 67.20 & 23.89 & 24.10 & 23.93 \\
\hline Macromolecule & 35.82 & 67.17 & 23.53 & 23.53 & 23.06 \\
\hline Ligand & 28.07 & 81.58 & 15.94 & 17.65 & 16.64 \\
\hline Solvent & 42.87 & 58.02 & 29.39 & 30.62 & 31.29 \\
\hline PDB ID & $6 \mathrm{~S} 19$ & 6RVM & $6 \mathrm{RVN}$ & 6RVP & $6 \mathrm{RVQ}$ \\
\hline
\end{tabular}

Statistics for the highest-resolution shell are shown in parentheses 


\section{FIGURE LEGEND}

Figure S1 (related to Figure 1). Supporting $1 \mathrm{D}{ }^{1} \mathrm{H}$ NMR spectra of SaFtsZ in $10 \mathrm{mM}$ dTris (D11, 98\%), $50 \mathrm{mM} \mathrm{KCl}$ buffer in $\mathrm{D}_{2} \mathrm{O}$ (100\%), $\mathrm{pH}^{*} 7.4,25.0{ }^{\circ} \mathrm{C} .431 \mu \mathrm{M} \mathrm{SaFtsZ}$ f plus $1 \mathrm{mM}$ GTP (red), $600 \mu \mathrm{M} \mathrm{SaFtsZ}$ T plus $1 \mathrm{mM}$ GTP (green), $260 \mu \mathrm{M}$ apo-SaFtsZ T $_{\mathrm{T}}$ without GTP (black) and after adding $1 \mathrm{mM}$ GTP (light green).

Figure S2 (related to Figure 1): Supporting $1 \mathrm{D}{ }^{1} \mathrm{H}$ NMR spectra recorded on SaFtsZ $\mathrm{T}_{\mathrm{T}}$ (panel A) or SaFtsZ $\mathrm{f}_{\mathrm{f}}$ (panel B) in $1.0 \mathrm{mM} \mathrm{GTP,} 10 \mathrm{mM}$ Tris (D11, 98\%), $50 \mathrm{mM} \mathrm{KCl,} \mathrm{with} 0.20 \mathrm{mM} \mathrm{DSS}$ as the internal chemical shift reference. The samples had been incubated with GTP in $\mathrm{H}_{2} \mathrm{O}$ buffer prior to transfer to $\mathrm{D}_{2} \mathrm{O}$ buffer. The spectra were recorded at $5.0^{\circ} \mathrm{C}$ (black), $15.0{ }^{\circ} \mathrm{C}$ (blue), 25.0 ${ }^{\circ} \mathrm{C}$ (green), $35.0{ }^{\circ} \mathrm{C}$ (red) and $45.0{ }^{\circ} \mathrm{C}$ (magenta).

Figure S3 (related to Figure 3). Supporting CD spectra of $20 \mu \mathrm{M}$ apo-SaFts $\mathrm{T}_{\mathrm{T}}$ with varying $\mathrm{KCl}$ concentrations in $10 \mathrm{mM}$ Tris-HCl, pH 7.5, $25^{\circ} \mathrm{C}$. Black, $50 \mathrm{mM} \mathrm{KCl}$; blue, $100 \mathrm{mM}$; light blue, 150 mM; dark green, 200 mM; green 250 mM; dark pink, 300 mM; pink, 375 mM; red, $450 \mathrm{mM}$; brown, $600 \mathrm{mM}$. The inset shows the change in ellipticity at $222 \mathrm{~nm}$ as a function of $\mathrm{KCl}$ concentration. The line is a least-squares fit to a two-state model (Methods) with $\Delta \mathrm{G}_{\text {fold, } 0 \mathrm{M}}$ $\mathrm{KCl}=3.6 \pm 0.8 \mathrm{kcal} \mathrm{mol}^{-1}$, additive efficacy $m=12.58 \pm 2.82 \mathrm{kcal} \mathrm{mol}^{-1} \mathrm{M}^{-1}$, ellipticity (min) = $3130 \pm 290$, ellipticity $(\max )=-9640 \pm 450, r^{2}=0.984$, end-state slope $s=-1200 \pm 480$. All CD spectra were acquired after $120 \mathrm{~min}$ incubation of the protein in the corresponding buffer.

Figure S4 (related to Figure 3). Supporting CD spectra of $20 \mu \mathrm{M}$ apo-SaFtsZ $\mathrm{T}_{\mathrm{T}}$ with various additives at $25^{\circ} \mathrm{C}$. A. Effects of $30 \%$ (v/v) glycerol, same plus $450 \mathrm{mM} \mathrm{KCl,} 200 \mathrm{~g} / \mathrm{L}$ Ficoll and

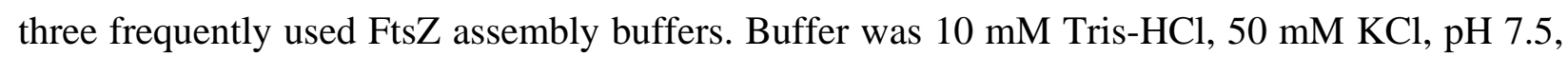
except where indicated. MES: $50 \mathrm{mM}$ Mes, $50 \mathrm{mM} \mathrm{KCl,} 1 \mathrm{mM}$ EDTA, $10 \mathrm{mM} \mathrm{MgCl}$, pH 6.5 (assembly buffer, Fig. 2). HMK: 50 mM Hepes, 100 mM KAc, 5 mM MgAc, 1 mM EGTA, pH 7.7 [69]. MES200: 100 mM Mes, $200 \mathrm{mM} \mathrm{KCl,} 5$ mM MgCl 2,1 mM EGTA, pH adjusted to 6.5 [8]. The apo-SaFtsZ spectrum from Fig. 4A is shown here for comparison. B. Compared effects of 25\% (v/v) glycerol, 20\% (v/v) ethylene glycol, 20\% (v/v) methyl-pentane diol, 1M TMAO, getaine, proline or trehalose. All CD spectra were acquired after 120 min incubation of the protein in the corresponding buffer.

Figure S5 (related to Figure 3): Supporting $1 \mathrm{D}{ }^{1} \mathrm{H}$ NMR spectra during GTP-Induced Refolding of SaFtsZ $\mathrm{T}$ at $25.0^{\circ} \mathrm{C}$ in $10 \mathrm{mM}$ TRIS (D11, 98\%), $50 \mathrm{mM} \mathrm{KCl,} 0.20 \mathrm{mM}$ DSS, 100 $\% \mathrm{D}_{2} \mathrm{O}, \mathrm{pH}^{*}$ 7.4. A. Complete spectra acquired before GTP addition (gray dashed line); and 10.5 min (black), 32 min (blue), 59 min (cyan), 80 min (green) and 445 min (red) after adding GTP to 
a final concentration of $2.0 \mathrm{mM}$. The peak at $0.00 \mathrm{ppm}$ arises from the trimethyl moiety of DSS, the internal chemical shift reference. B. Zoomed view of the upfield spectral region. As time passes, the decreasing height and broadening of the peaks at $1.0-0.8 \mathrm{ppm}$, which arise from disordered methyl ${ }^{1} \mathrm{H}$, are notable. C. Zoomed view of the downfield spectral region. The intense, sharp peaks at 8.13 and 5.92 ppm arise from the GTP H8 and H1', respectively. After adding GTP, a series of small peaks appear and grow in the regions 7.1 - 6.7 ppm and 6.1 - 5-, 1 ppm, which can be putatively attributed to Phe ring ${ }^{1} \mathrm{H}$ in specific conformations in the hydrophobic core and to ${ }^{1} \mathrm{H} \alpha$ which are hydrogen bonded in $\beta$-sheets, respectively. D. Highly magnified zoom of the upfield spectral region. Methyl ${ }^{1} \mathrm{H}$ over aromatic rings in the hydrophobic core give rise to the growing peaks observed at $0.64,0.48,0.26$ and $-0.13 \mathrm{ppm}$.

Figure S6 (related to Figure 5). Supporting structural data. A. Structures of $\mathrm{SaFts}_{\mathrm{T}}$ in the presence of GDP. Canonical front view of the three GDP-bound $\mathrm{SaFtsZ}_{\mathrm{T}}$ structures reported here (colored) superimposed to the previously reported structure PDB-ID 3VOA (grey). A blue star indicates a cavity in the GDP-binding pocket that is empty in SaFtsZ-Wat (yellow), while it contains 1-methyl-2-pyrrolidone or ethylene glycol in SaFtsZ-MetPyr (purple) or SaFtsZ-EthGly (green). Differences in the superposition are shown with arrows and distances. These three SaFtsZ $_{\mathrm{T}}$ structures are nearly identical, with alpha carbon rmsd values of $0.18-0.28 \AA$ among them and with refolded SaFtsZ $\mathrm{T}_{\mathrm{T}}$ (Figure 5). They can be superposed to equivalent available structures with similar rmsd values (0.25-0.29, 0.27-0.34 and 0.28-0.33 $\AA$ for PDB entries 3VOA (40), 5H5G (41) and 5MN4 (20), respectively). In the structures reported here, the N-terminal extension derived from the cloning strategy is shifted with respect to 3VOA, likely due to different sequences between them (GSHM in our structures versus -GHM in 3VOA and 5H5G) and with wild-type SaFtsZ (FNHL). This extension, which is absent from the construct in 5MN4, lies nearby the T3 loop of the next monomer. Accordingly, the conformation of T3 also presents minor differences between the structures reported here and 3VOA. B. A cavity behind the NBS is occupied by six water molecules in SaFts $\mathrm{Z}_{\mathrm{T}}$-Wat, 1-methyl-2-pyrrolidone in SaFtsZ $\mathrm{T}_{\mathrm{T}}-\mathrm{MetPyr}$, or ethylene glycol in SaFtsZ $\mathrm{T}_{\mathrm{T}}$-EthGly. C. Chemical structures of molecules seen in the cavity in structures reported here or elsewhere [10] and (right) SaFtsZ residues lining the cavity, involved in ligand contacts.

Figure S7 (related to Figure 5). Supporting structure of the $\mathrm{SaFts}_{\mathrm{T}}$ folded apo state in ammonium sulfate. A. The four molecules in the asymmetric unit of the crystals are shown in different colors, superimposed to the previously reported structure PDB-3VPA (grey). Sulfate ions and glycerol molecules are shown as spheres, while the neighboring arginine residues of the 
protein are shown as sticks. B. Superposition of the four molecules in the asymmetric unit, using colors as in panel A. C. Superposition as in panel B including the 4 molecules of the previously reported structure PDB-3VPA in light colors. The T3 loop (residues 67-74) and T4 loop (residues 105-109) reveal significant variability between the molecules, while the T5 loop (residues 135-140) is only fully ordered in one of the molecules. D. Conformational changes from the GDP-bound and folded apo states, indicated with arrows. The view is $90^{\circ}$ rotated from the canonical front view shown in Figure 3, with only the twist region shown. See Movie 1.

Figure S8 (related to main text). Data from a screen of different additives for high affinity

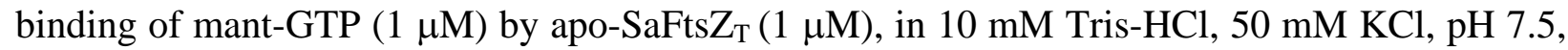
$25{ }^{\circ} \mathrm{C}$. Binding was monitored by the fluorescence anisotropy of mant-GTP. The anisotropy value of free mant-GTP was subtracted in each condition; the increment anisotropy plotted was confirmed specific by addition of an excess of GTP. Additive concentrations are indicated on the upper and lower X-axis. The chemical structures of the interconverting mant-GTP isomers are shown below.

Figure S9 (related to Figure 6). A. Titration data of $500 \mathrm{nM}$ mant-GTP with apo-SaFtsZ $\mathrm{T}_{\mathrm{T}}$ in 10 mM Tris, 50 mM KCl, 1 mM EDTA, pH 7.5, 3.4 M glycerol, 2\% DMSO at $25{ }^{\circ} \mathrm{C}$, measuring fluorescence intensity. The ratio between the fluorescence intensities of SaFts $\mathrm{Z}_{\mathrm{T}}$-bound and free mant-GTP determined from this titration $(\mathrm{R}=2.8)$ was used to correct all anisotropy-based binding calculations. B. Same titration as in panel A, employing mant-GTP fluorescence anisotropy to determine the fraction of ligand bound by the protein, which gave an equilibrium dissociation constant $\mathrm{K}_{\mathrm{D}}=570 \pm 80 \mathrm{nM}$ and anisotropy values for free mant-GTP $\mathrm{r}_{\text {free }}=0.021 \pm$ 0.006 and bound mant-GTP $r_{\text {bound }}=0.223 \pm 0.005$. Lines in each case correspond to best leastsquares fits to the data.

Movie 1 (related to main text and Figure 5). Morph between the crystal structures of SaFts $\mathrm{Z}_{\mathrm{T}}$ from the GDP-bound state and the folded apo state. SaFtsZ $\mathrm{T}_{\mathrm{T}}$ is shown in the canonical view, with the nucleotide-binding domain in cyan, the C-terminal domain in gold, and the twist region in gray. The main structural elements in each state have been labeled. 
Figure S1

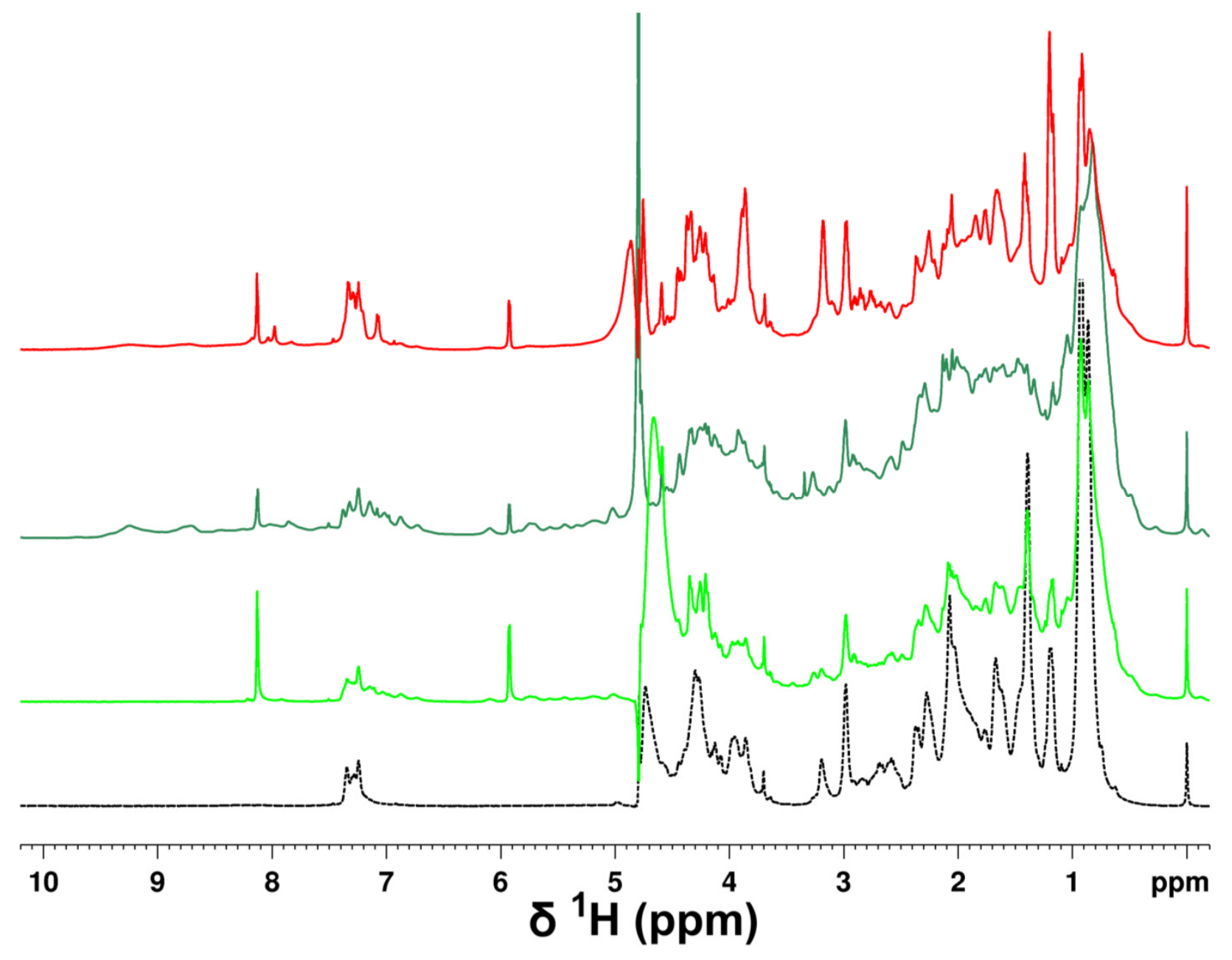


Figure S2
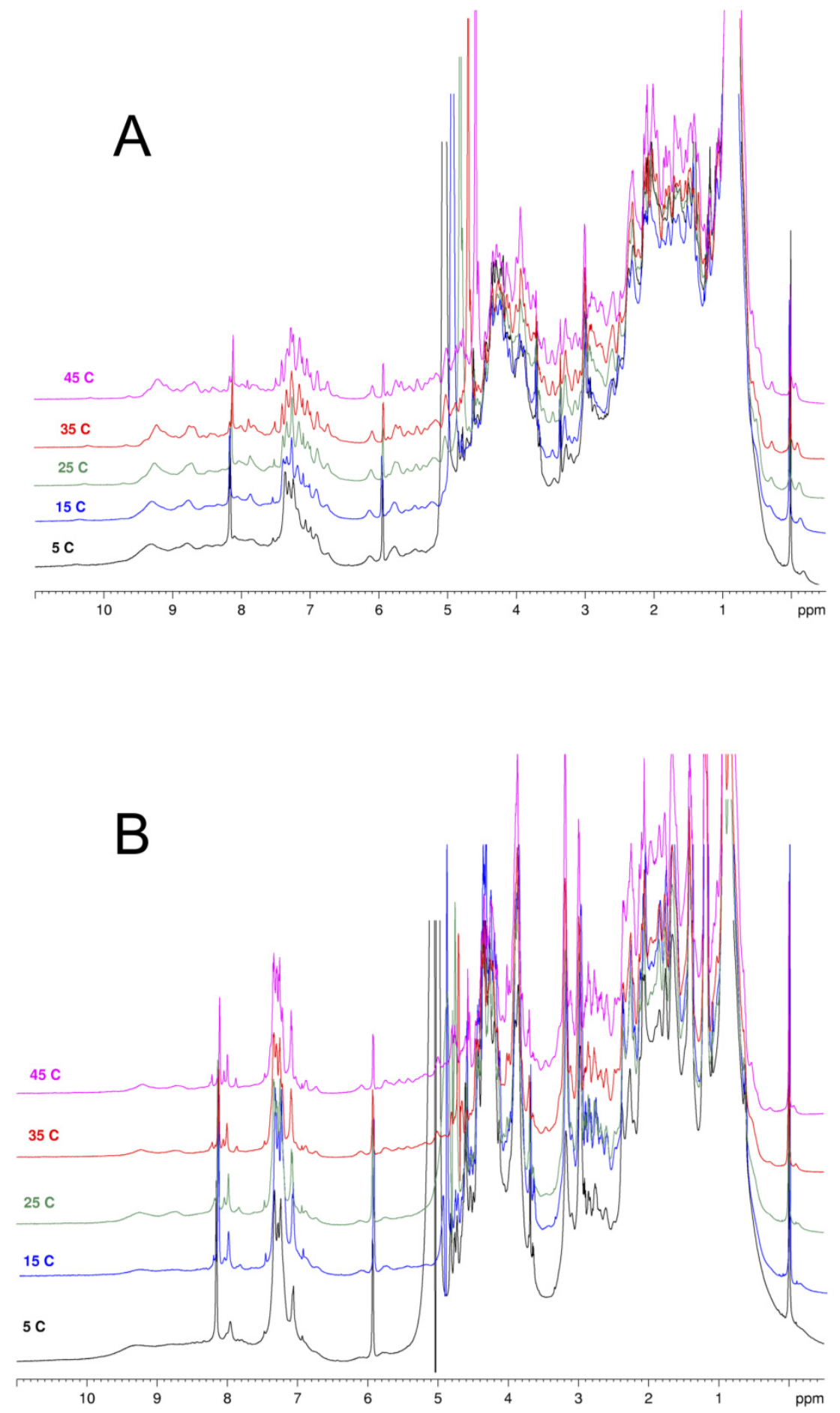
Figure S3

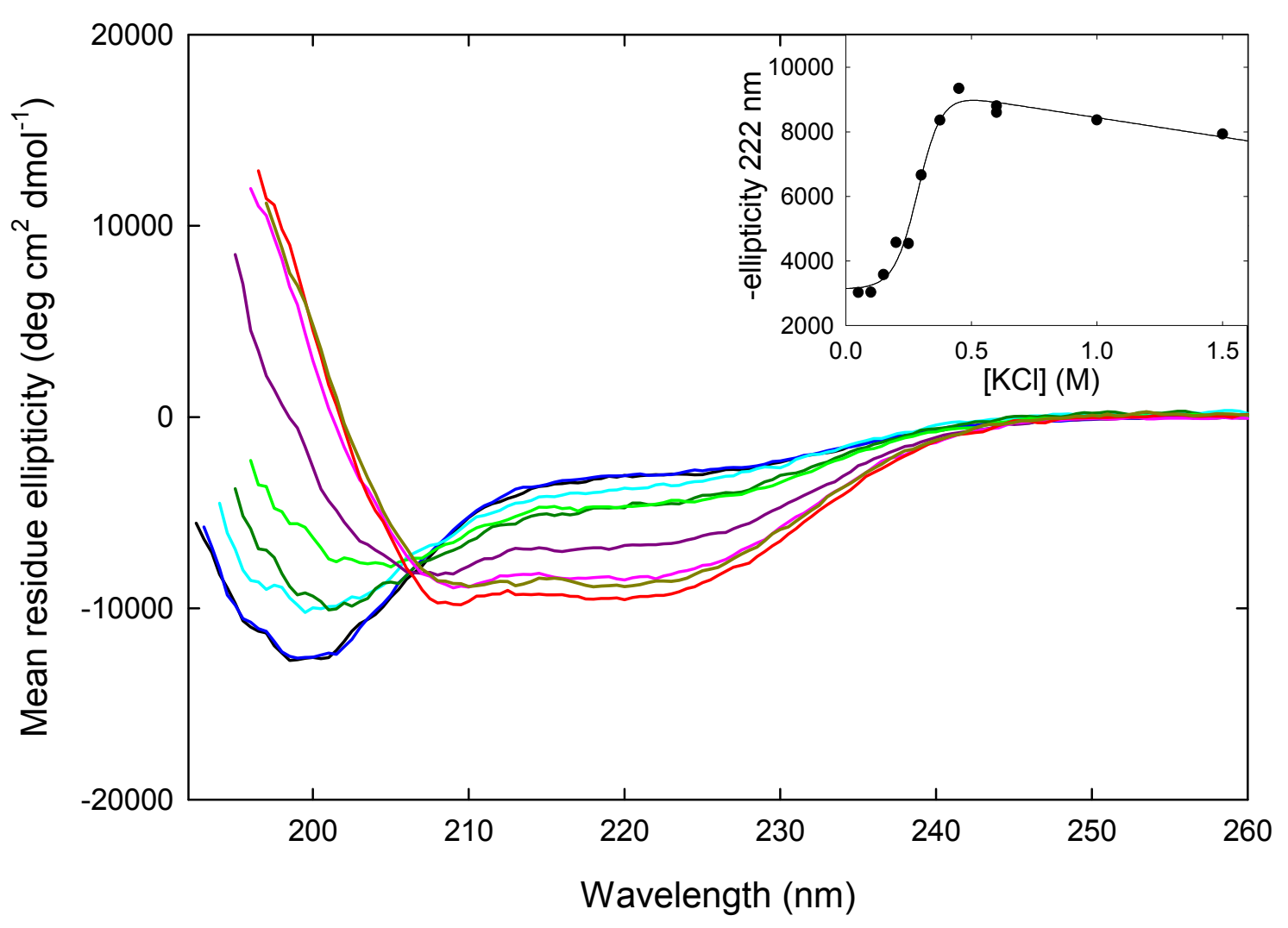


Figure S4

A
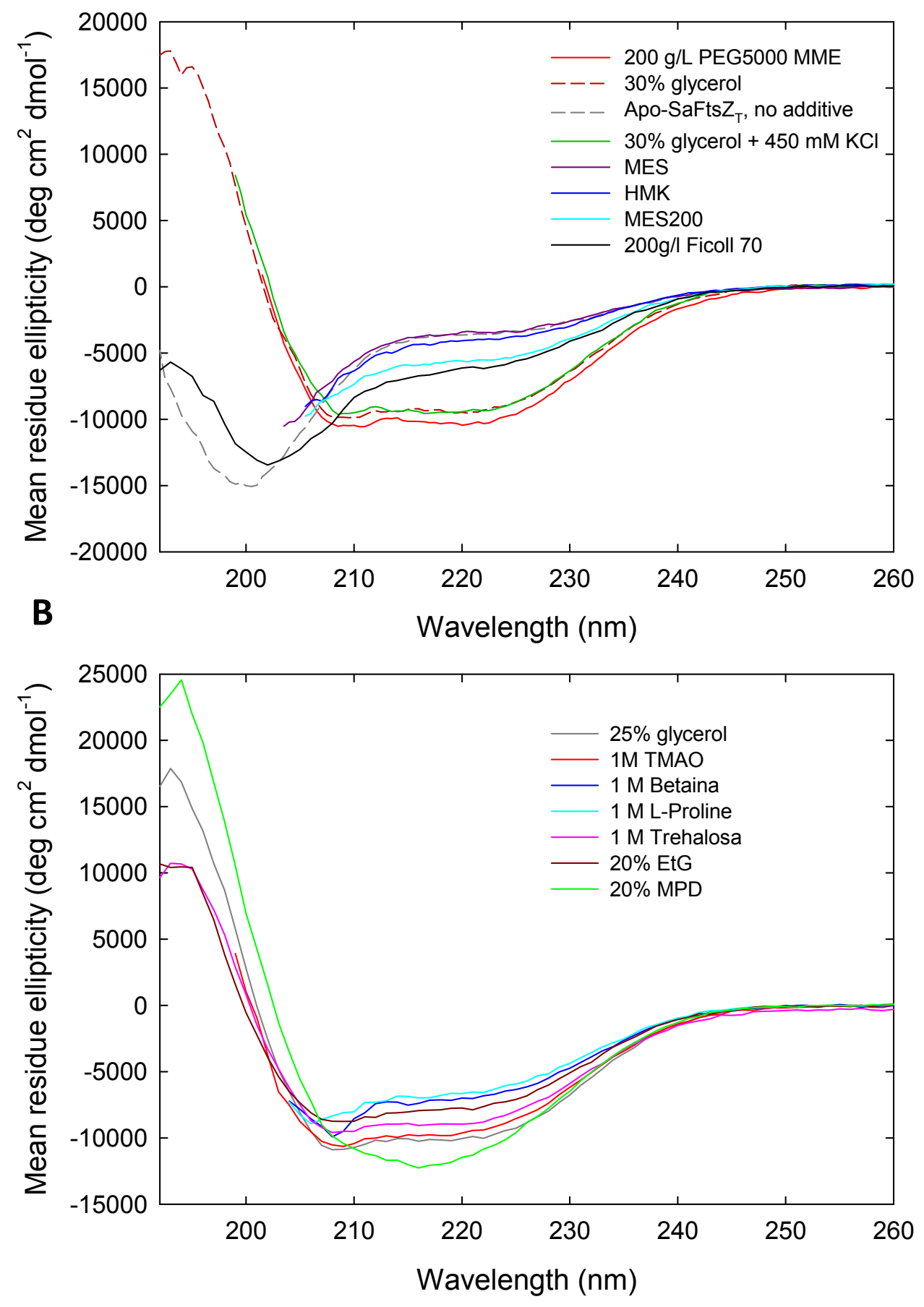
Figure S5
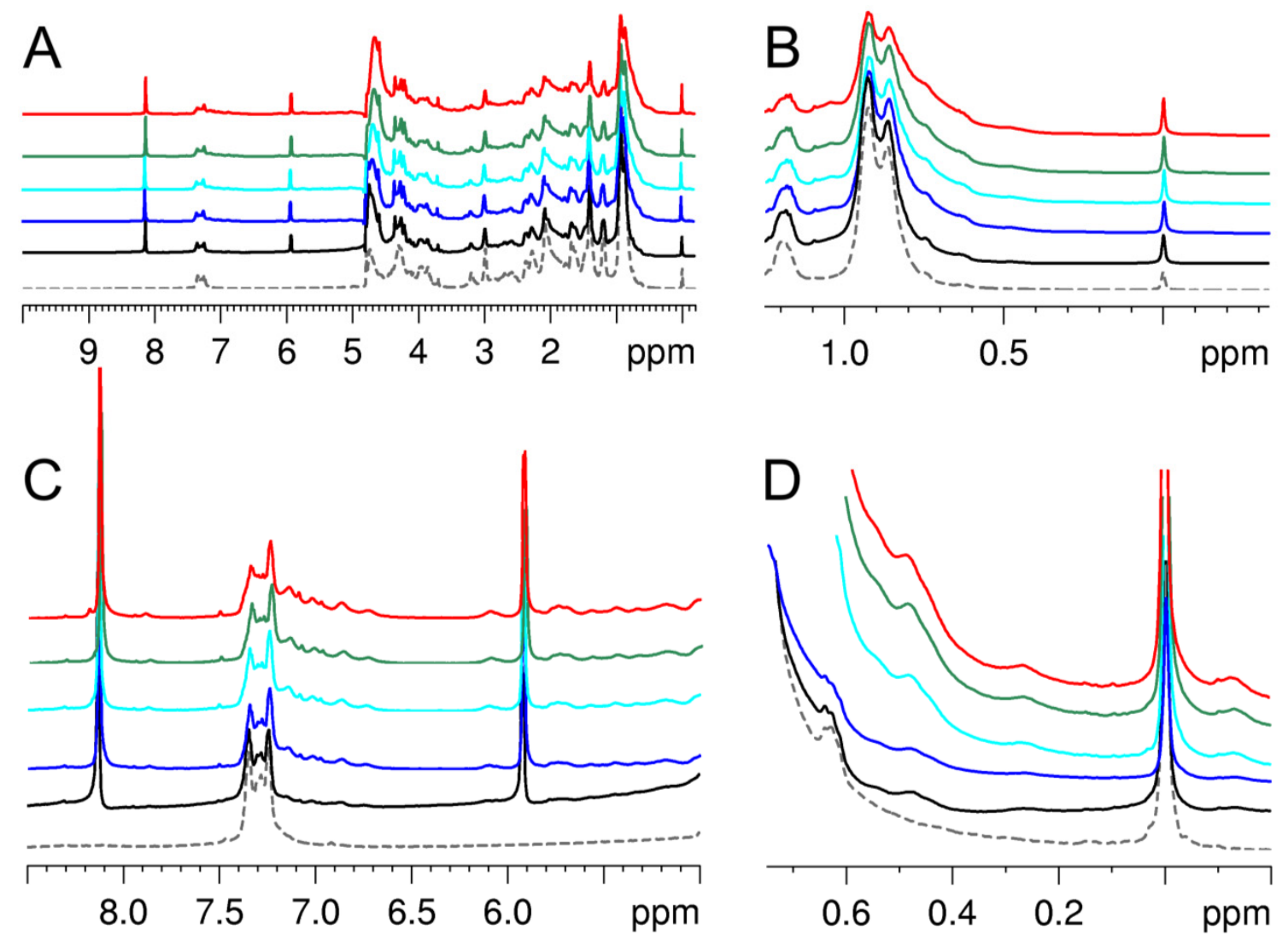
Figure S6

A

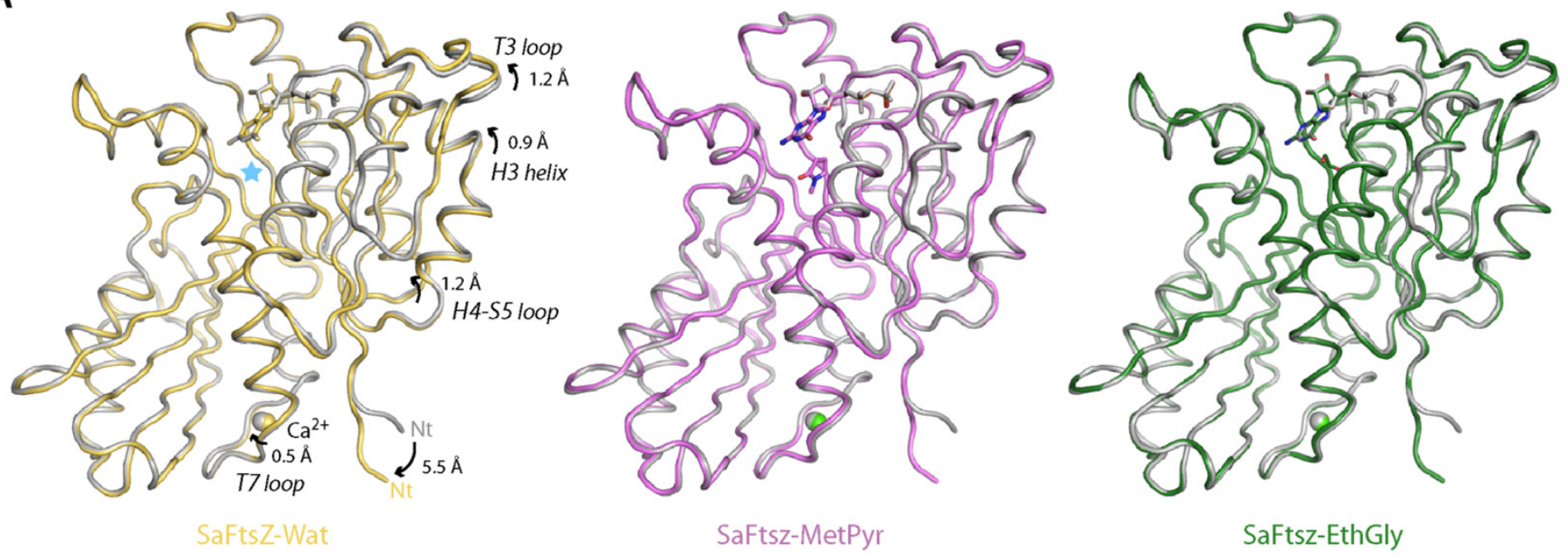

B

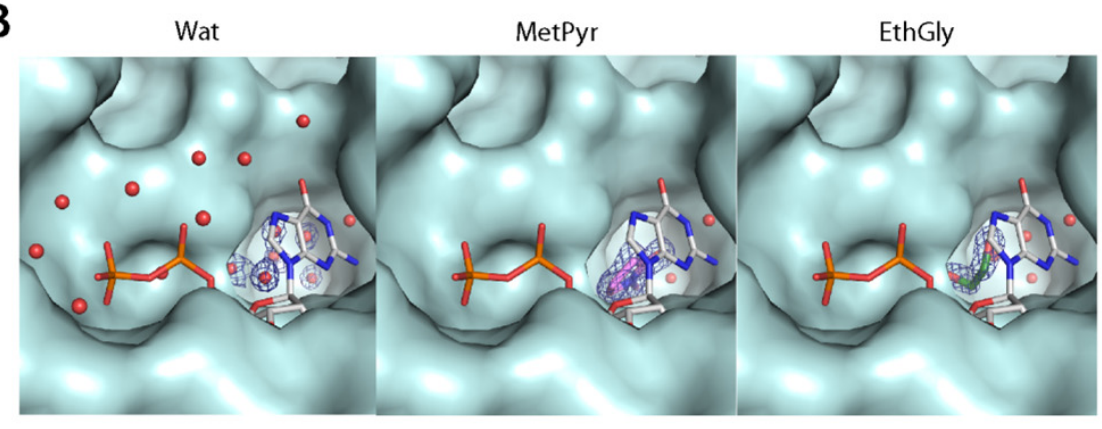

C

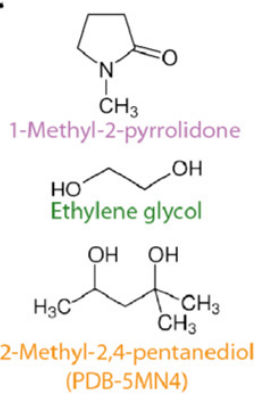

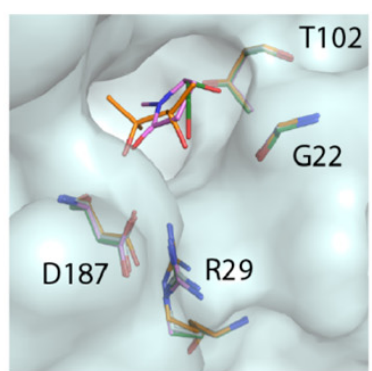


Figure S7
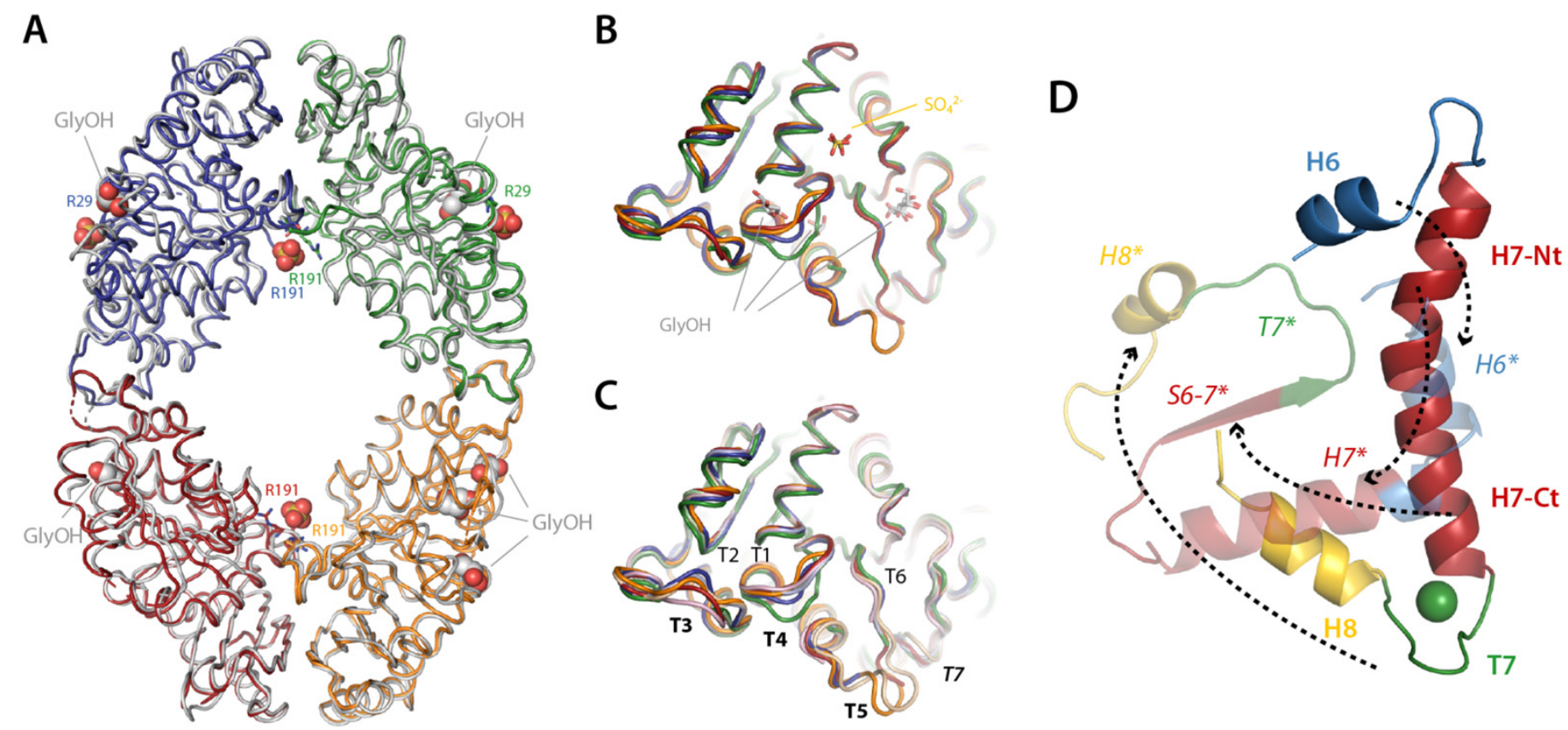


\section{Figure S8}

[TMAO, trehalose, betaine, L-proline, $\mathrm{KCl}, \mathrm{KAc}, \mathrm{KGlu}, \mathrm{NaGlu}$ ] (M)

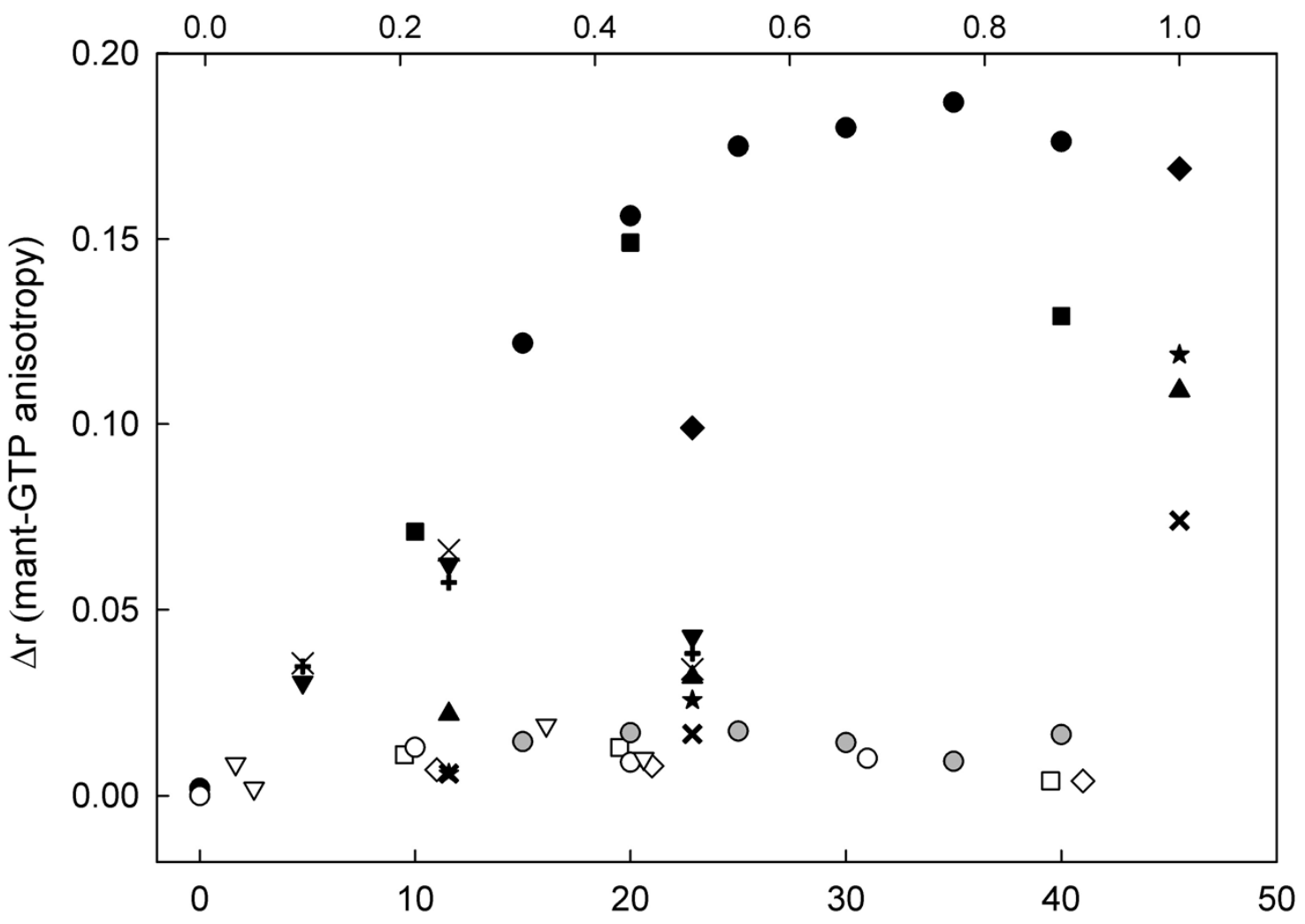

[Glycerol, ethyleneglycol, MPD] (\% v/v), [PEG 5000, Ficoll 70] (\% w/v)
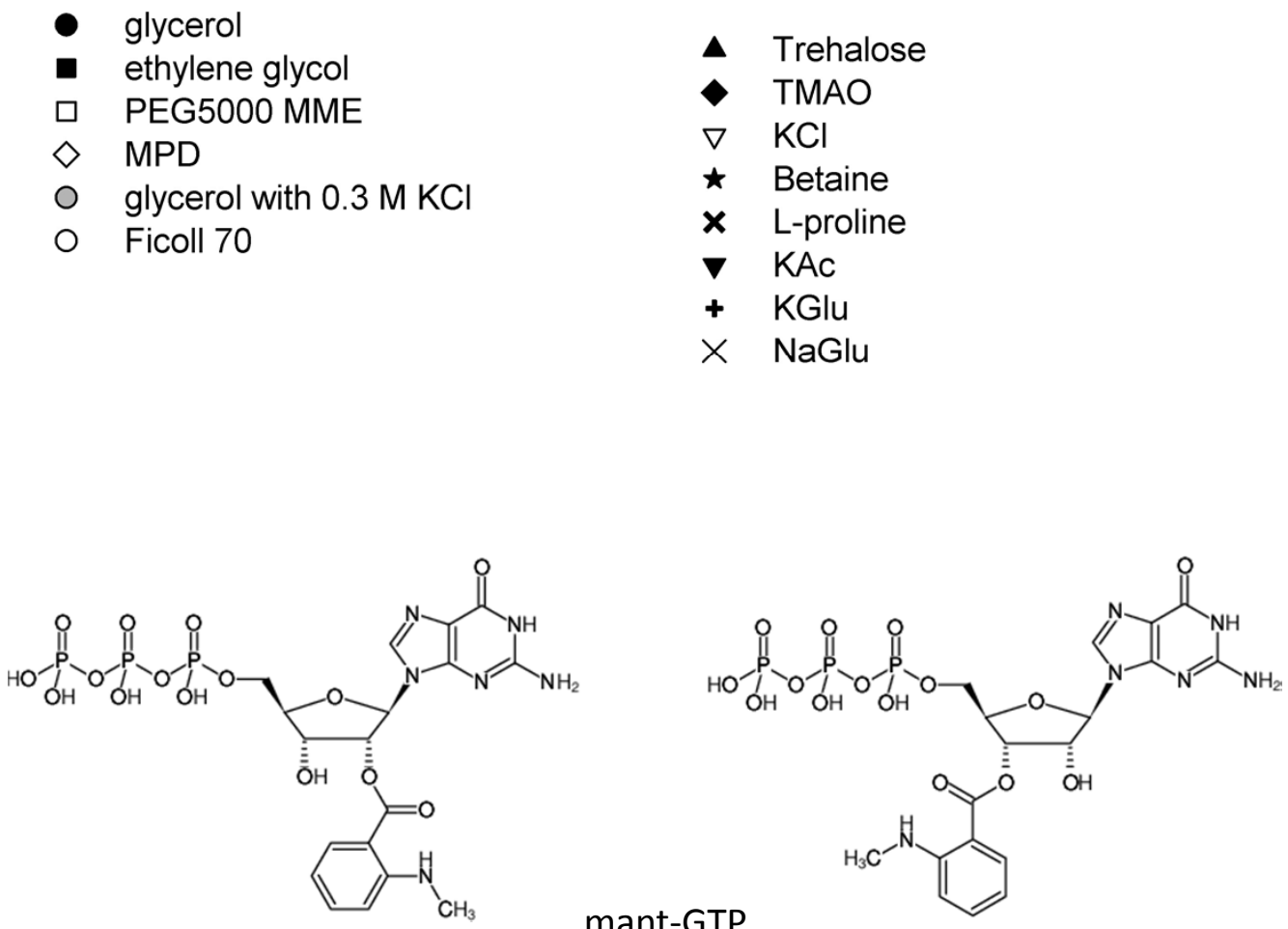

mant-GTP 
Figure S9
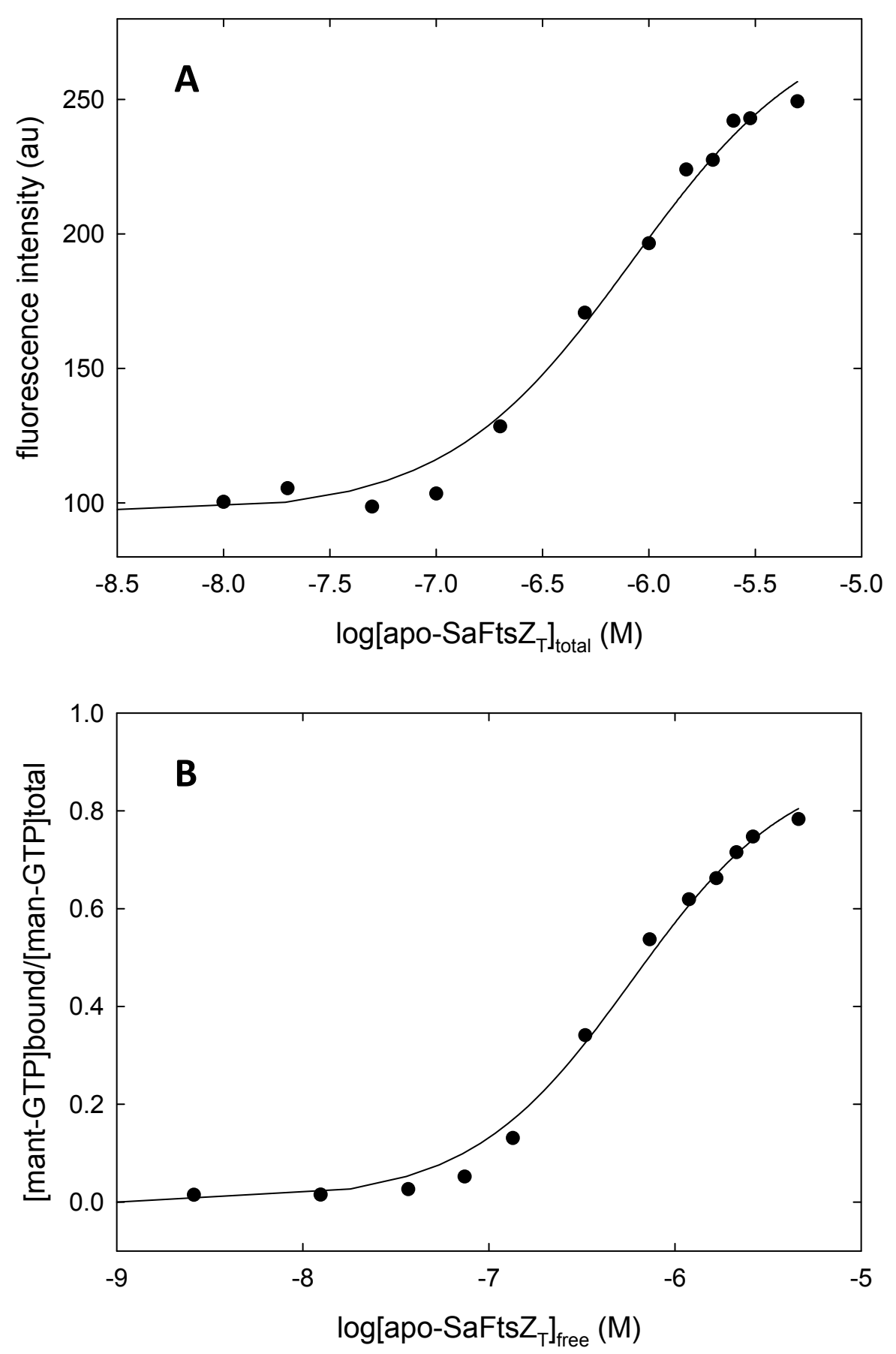